\title{
Bihemispheric Transcranial Direct Current Stimulation Enhances Effector-Independent Representations of Motor Synergy and Sequence Learning
}

\author{
Sheena Waters-Metenier, ${ }^{1,2}$ Masud Husain, ${ }^{1,2,3,4}$ Tobias Wiestler, ${ }^{1}$ and Jörn Diedrichsen ${ }^{1}$ \\ Institutes of ${ }^{1}$ Cognitive Neuroscience and ${ }^{2}$ Neurology, University College London, London WC1N 3AR/WC1N 3BG, United Kingdom, and ${ }^{3}$ Nuffield \\ Department of Clinical Neurosciences and ${ }^{4}$ Department of Experimental Psychology, Oxford University, Oxford OX3 9DU/OX1 3UD, United Kingdom
}

Complex manual tasks - everything from buttoning up a shirt to playing the piano-fundamentally involve two components: (1) generating specific patterns of muscle activity (here, termed "synergies"); and (2) stringing these into purposeful sequences. Although transcranial direct current stimulation (tDCS) of the primary motor cortex (M1) has been found to increase the learning of motor sequences, it is unknown whether it can similarly facilitate motor synergy learning. Here, we determined the effects of tDCS on the learning of motor synergies using a novel hand configuration task that required the production of difficult muscular activation patterns. Bihemispheric tDCS was applied to M1 of healthy, right-handed human participants during $4 \mathrm{~d}$ of repetitive left-hand configuration training in a double-blind design. tDCS augmented synergy learning, leading subsequently to faster and more synchronized execution. This effect persisted for at least 4 weeks after training. Qualitatively similar tDCS-associated improvements occurred during training of finger sequences in a separate subject cohort. We additionally determined whether tDCS only improved the acquisition of motor memories for specific synergies/sequences or whether it also facilitated more general parts of the motor representations, which could be transferred to novel movements. Critically, we observed that tDCS effects generalized to untrained hand configurations and untrained finger sequences (i.e., were nonspecific), as well as to the untrained hand (i.e., were effector-independent). Hence, bihemispheric tDCS may be a promising adjunct to neurorehabilitative training regimes, in which broad transfer to everyday tasks is highly desirable.

Key words: tDCS; motor cortex; learning; generalization; motor synergy; motor sequence

\section{Introduction}

The acquisition of fine hand motor skills requires two types of learning: (1) how to generate novel patterns of muscular activity (synergies); and (2) how to properly sequence them. The latter element-sequence learning-is well described behaviorally (Dayan and Cohen, 2011; Penhune and Steele, 2012) and is associated with functional (Hardwick et al., 2013) and structural (Steele et al., 2012) changes in motor regions, particularly primary motor cortex (M1). Comparatively, much less is known about the principles underlying synergy learning.

A long-standing supposition in motor control is that the nervous system generates movement through synergic muscle

\footnotetext{
Received May 28, 2013; revised Nov. 18, 2013; accepted Nov. 24, 2013.

Author contributions:S.W.-M., M.H., T.W., and J.D. designed research;S.W.-M. performed research;S.W.-M. and J.D. analyzed data; S.W.-M., M.H., and J.D. wrote the paper.

The research was supported by Brain Research Trust Grant 6CHB (S.W.-M.) and Wellcome Trust Grant 094874/Z/ 10/Z (J.D.). We thank Alexandra Reichenbach and Joseph Galea for advice regarding TMS and tDCS and Naveed Ejaz and George Prichard for comments on this manuscript.

The authors declare no competing financial interests.

This article is freely available online through the J Neurosci Author Open Choice option.

Correspondence should be addressed to Sheena Waters-Metenier, Institute of Cognitive Neuroscience, University

College London, 17 Queen Square, London, WC1N 3AR, UK. E-mail: sheena.waters@gmail.com.

DOI:10.1523/JNEUROSCI.2282-13.2014

Copyright $\odot 2014$ Waters-Metenier et al.

This is an Open Access article distributed under the terms of the Creative Commons Attribution License (http://creativecommons.org/licenses/by/3.0), which permits unrestricted use, distribution and reproduction in any medium provided that the original work is properly attributed.
}

groupings, which simplify control (Sherrington, 1910; Bizzi et al., 2002). Note that we use the term "synergy" here in a purely descriptive sense to refer to frequently co-occurring spatiotemporal patterns of muscle activity across movements, independently of how they may arise from neural control structures (Tresch and Jarc, 2009; Diedrichsen et al., 2010). One hypothesis regarding the neural implementation of synergies is that frequently generated muscle activity patterns are encoded in a modular manner in M1. Evidence of such architecture has been provided by microstimulation in macaques (Overduin et al., 2012) and transcranial magnetic stimulation (TMS) in humans (Gentner and Classen, 2006), which evoke muscle activity patterns that resemble the principle features of natural or trained (Gentner et al., 2010) movements. Under this premise, learning to produce a novel muscle activity pattern would entail formation of a dedicated neural control module encoding this synergy. This would enable more efficient execution, because activation of a single neural module—rather than multiple—is required.

We studied this learning process through a novel hand configuration task and determined whether synergy learning could be modulated by bihemispheric transcranial direct current stimulation (tDCS) of M1. Through the induction of weak intracerebral ionic current between a positively charged anode and negatively charged cathode, tDCS elicits regional- and polarityspecific modification of neural excitability (Priori et al., 1998), and, putatively, neural plasticity (Fritsch et al., 2010). M1 tDCS 
facilitates the learning of sequential pinch forces (Reis et al., 2009) — a task involving only one muscle synergy (between thumb and index finger) - but whether it influences synergy learning is unexplored. This question is of considerable practical importance, because a key feature of motor dysfunction involving corticospinal lesions (e.g., after stroke) is impaired finger individuation (Lawrence and Kuypers, 1968; Lang and Schieber, 2003, 2004; Krakauer, 2005), which is likely driven by reduction of functional synergies (Cheung et al., 2012).

Additionally, we characterized the locus of tDCS effects on synergy learning by quantifying generalization to unpracticed hand configurations and the untrained hand. If tDCS accelerates the formation of specific, effector-dependent representations (e.g., dedicated control modules in M1), then tDCS effects would generalize poorly to new hand configurations and the untrained hand. However, if tDCS also enhances the formation of nonspecific, effector-independent representations of skill, then the stimulation-associated performance gains should generalize broadly to untrained configurations and the untrained hand. Finally, we qualitatively compared tDCS-related changes in synergy learning and generalization with tDCS effects observed with conventional finger sequence training.

\section{Materials and Methods}

\section{Participants}

Fifty-two normal, healthy right-handed human subjects (27 males; mean age, $21.90 \pm 0.40$ years) participated in this study. Ethical permission was acquired from the University College London Research Ethics Committee. Exclusion criteria for participation were as follows: (1) age above 30 or below 18 years; (2) history of medical, neurological, or psychiatric illness; (3) personal/familial history of epilepsy or seizures; (4) current usage of drugs known to influence behavior or cognition; (5) recent $(<3$ months) exposure to brain stimulation; (6) disability of the fingers, hands, or wrist; and (7) significant experience with musical instruments or computer gaming ( $>1000 \mathrm{~h}$ of practice). All participants gave written informed consent in accordance with the Declaration of Helsinki (World Medical Association, 2002) and were provided with an honorarium for participation. Participants were assigned to one of two cohorts: 28 trained on a hand configuration (synergy learning) task and the other 24 trained on a finger sequence task. Each training cohort was divided into two stimulation groups: half of the participants received tDCS and the remainder received sham.

At the beginning of the experiment, subjects completed the Edinburgh Handedness Inventory (Oldfield, 1971), as well as a survey encompassing medical history, musical and computer gaming experience, and previous exposure to brain stimulation methodologies. Additionally, during all sessions, participants provided information about sleep quality, alertness, attention, and task difficulty using visual analog scales ranging from 0 (lowest) to 10 (highest). There were no significant differences between tDCS and sham groups in terms of these parameters or demographics (Table 1).

\section{$t D C S$}

tDCS was administered via a bihemispheric montage, which may elicit larger behavioral improvements than conventional unihemispheric tDCS (Vines et al., 2008b). The anode was positioned over right M1 (which was contralateral to the trained left hand), and the cathode was placed above left (ipsilateral) M1. Using single-pulse suprathreshold TMS with a figure-of-eight coil of $5 \mathrm{~cm}$ diameter (Magstim BiStim2; Whitland) positioned tangentially to the skull at a $45^{\circ}$ angle, we localized right and left M1s by identifying the optimal scalp positions for activation of left and right first dorsal interosseus muscles (Boroojerdi et al., 1999). The optimal scalp positions were then delineated using a black permanent marker for electrode placement. Using a neuroConn DC-stimulator PLUS (http://www.neuroconn. de/dc-stimulator_plus_en/), tDCS was applied at an intensity of $2.0 \mathrm{~mA}$ (current density, $0.057 \mathrm{~mA} / \mathrm{cm}^{2}$ ) through two flat carbon electrodes (surface area, $35 \mathrm{~cm}^{2}$ ) encased in saline-soaked sponges, which were secured by adhesive Coban $3 \mathrm{M}$ bandages.

Participants were assigned to tDCS and sham groups in a pseudorandomized manner contingent on pretest performance. Specifically, the first two participants were designated randomly, and all subsequent individuals were assigned such that the between-group mean difference in baseline performance was minimized. To keep both participants and the test administrator (S. Waters-Metenier) blind to stimulation group assignment, J. Diedrichsen generated a unique neuroConn preset code for every participant, which was entered into the machine at the beginning of training.

Twenty-five minutes of stimulation was delivered to tDCS recipients, whereas sham involved brief stimulation (15 s) with subsequent impedance monitoring. For both groups, the current was increased over $10 \mathrm{~s}$ and diminished over $10 \mathrm{~s}$ via a ramped technique. Similar sham conditions appear to be sufficient to blind participants in studies of $1 \mathrm{~mA}$ unihemispheric M1 tDCS, because the short period of stimulation elicits similar cutaneous sensations as real tDCS (Gandiga et al., 2006) — such as mild burning, pruritus, and paresthesia-which persist even after the ramp down phase (Ambrus et al., 2012). However, because blinding success has been inconsistent at intensities exceeding $1 \mathrm{~mA}$ (Ambrus et al., 2012; Kessler et al., 2012; O’Connell et al., 2012; Brunoni et al., 2013; Palm et al., 2013; Russo et al., 2013), we carefully monitored the perceptual properties associated with $2 \mathrm{~mA}$ bihemispheric tDCS using visual analog scales (as described previously).

There were no significant differences in discomfort, perceived tDCS intensity, or distraction between groups, although tDCS recipients tended to report slightly higher values on these measures (note that ratings of both sham and tDCS groups were low, $\leq 4$ of a possible rating of 10 in all cases) (Table 1). At the end of training, we asked participants whether they had received real tDCS. We then used a $\chi^{2}$ goodness-of-fit test to probe whether the probability of responding "yes" differed between the groups. For the configuration-trained cohort, the proportion of yes responses was higher in the sham group and, for the sequencetrained cohort, the proportion of yes responses was higher in the tDCS group; however, none of these differences were significant (Table 1). Collectively, these observations suggest that sham stimulation yielded adequate participant blinding.

In addition to monitoring perceptual differences, we quantified potential emotional consequences of tDCS by administering the Positive and Negative Affect Schedule (PANAS) (Watson et al., 1988) before and after each training session. We observed no differences between sham and tDCS groups in pretraining or posttraining positive or negative scores (Table 1), which indicates an absence of such side effects and corroborates previous findings (Reis et al., 2009; Plazier et al., 2012).

Because daily tDCS appears to be more effective than dispersed applications (Boggio et al., 2007; Alonzo et al., 2012), stimulation was administered for 4 consecutive training days. Additionally, because there is strong evidence that concurrent tDCS and behavioral training improve motor learning (Reis et al., 2009; Reis and Fritsch, 2011; Stagg et al., 2011) and that cortical excitability changes outlast stimulation (Nitsche and Paulus, 2001), tDCS was initiated at the beginning of each 60 min training session, and, after stimulation ended, participants continued practicing for $\sim 35 \mathrm{~min}$. Subjects were always tested at the same time of day $( \pm 1 \mathrm{~h})$.

\section{Apparatus and stimuli}

All tasks were performed on a custom-built keyboard with $1010.5 \times 2$ $\mathrm{cm}$ keys. The piano-like keys were stationary and featured indentations in which the fingertips could be securely placed (Wiestler et al., 2011). Participants were seated in front of the apparatus to yield a position similar to that used in piano playing. Forces were measured every $5 \mathrm{~ms}$ via transducers (FSG-15N1A; Sensing and Control Honeywell; dynamic range of $0-25 \mathrm{~N}$, repeatability of a constant force $<0.02 \mathrm{~N}$ ), located beneath the fingertip indentation of each button. For all tasks, $101-\mathrm{cm}-$ long white lines-hereafter referred to as "force cursors"-were displayed on a $34 \times 27 \mathrm{~cm}$ computer screen such that the vertical position of 
Table 1. Demographic characteristics, baseline motor performance, and psychological measures

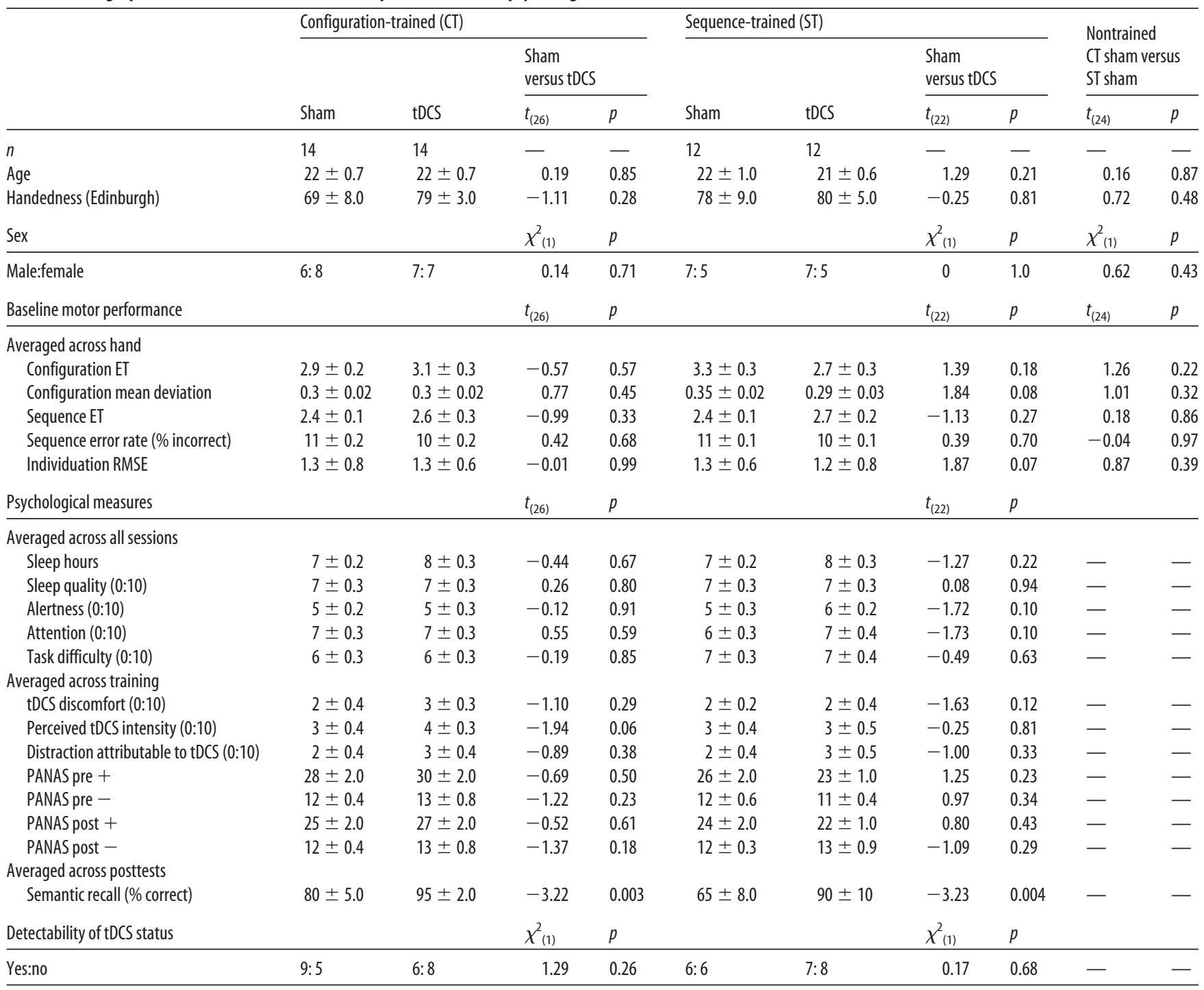

For each training cohort, $t$ tests are presented to compare the demographic, baseline motor performance, and psychological features of sham versus tDCS groups (negative $t$ indicates $t D C S>$ sham). However, to ascertain subject blindnes to tDCS group assignment, a $\chi^{2}$ goodness-of-fit test was used to probe whether the probability of responding yes to tDCS assignment differed between sham and tDCS groups (note that this statistic was also used for the categorical, binary variable sex). Additionally, demographic and performance differences between the configuration-trained (CT) and sequence-trained (ST) sham groups (which each served as the nontrained group of the respective other task) are reported. PANAS, Positive and Negative Affect Schedule ("pre" refers to PANAS measures before training and "post" to measures collected after training).

each line was proportional to the force exerted by each finger on the respective key.

\section{Procedure}

The primary aim of this study was to characterize the effects of bihemispheric M1 tDCS on learning and generalization of motor synergies. A secondary aim was to compare these effects with tDCSassociated changes in learning and generalization of motor sequences. Therefore, two different cohorts of participants (configuration-trained and sequence-trained) were trained with the nondominant left hand over four sessions (days 1-4) while receiving bihemispheric tDCS or sham. We also quantified generalization of tDCS effects to untrained configurations or sequences ("intratask" generalization) and to the untrained hand ("intermanual" generalization): during the pretest and three posttests conducted after tDCS-coupled training (on days 5, 12, and 33), participants performed two different types of configurations or sequencestrained and untrained - with each hand (Fig. 1). Finally, to determine the extent to which configuration and sequence training benefits transferred to an untrained task ("intertask" generalization), during the pretest and posttests, participants also completed a finger individuation task.

Both configuration- and sequence-trained cohorts also performed pretest and posttests on the respective other untrained task. The performance on the untrained task was taken as a measure of how much performance improved from the pretest to the posttest in the absence of task-specific training (i.e., attributable to practice effects of repeated testing). Thus, the configurationand sequence-trained cohorts served as "nontrained" active control groups for each other. The performance of these nontrained groups was used as a baseline from which to assess the amount of true intratask and intermanual generalization resulting from left-hand training. Note that we only used the data from the respective nontrained sham group, because receiving tDCS during the trained task could alter performance on the untrained task (e.g., increase intertask transfer).

Configuration task. The configuration task involved the recurrent production of difficult hand muscle activation patterns. Participants had to simultaneously press two or three digits (designated "active" fingers) in piano chord-like patterns while keeping other ("passive") fingers within a restricted resting force range of $0.24-1.20 \mathrm{~N}$ (Fig. 2A), which was represented on a computer screen as a gray "baseline box."

During a $2.7 \mathrm{~s}$ announcement phase, gray bars corresponding to the keyboard buttons turned green to specify active fingers of each hand configuration, and bars designating passive fingers remained gray (Fig. $2 A)$. Each trial initiated after the forces applied by active and passive fingers were within resting range and not separated by a difference larger 

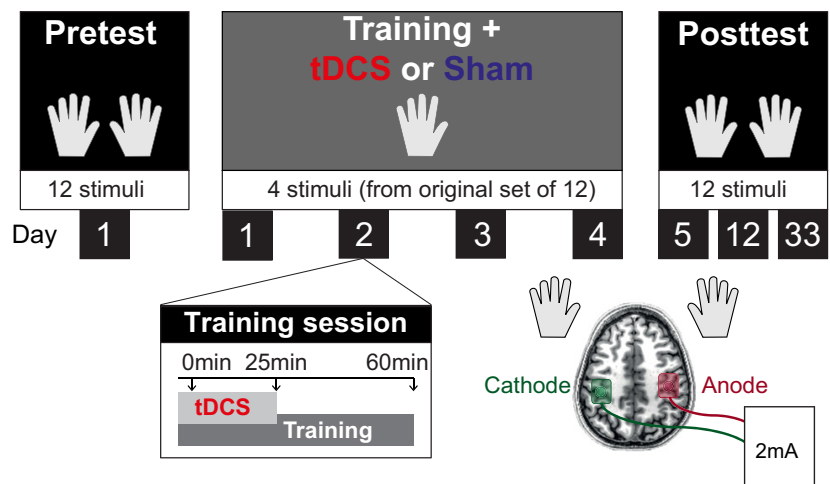

Figure 1. Study design. During the pretest, baseline performance of both hands was assessed for 12 unique stimuli (configurations or sequences). During the days of training, participants practiced four configurations or sequences repeatedly with the left hand and received real or sham $\mathrm{tDCS}$. Each $60 \mathrm{~min}$ training session began with $25 \mathrm{~min}$ of $2 \mathrm{~mA}$ bihemispheric tDCS or sham stimulation of M1. The anode (maroon) and cathode (green) were placed above M1 contralateral and ipsilateral to the trained left hand, respectively. During three posttests (days 5,12 , and 33), performance of both hands was again evaluated without tDCS. Finger individuation, as well as the respective other untrained task (i.e., the sequence task for the configuration-trained cohort and configuration task for the sequence-trained cohort) were additionally tested during pretest and posttests.

than $0.3 \mathrm{~N}$ for a period of $500 \mathrm{~ms}$ (note that this latter requirement prevented premature separation of active and passive finger forces-i.e., forming the configuration before the onset of the "go" signal while within the baseline force range-which is a strategy that could be used to increase task speed). A color change of the gray baseline box to green served as a go signal to specify trial commencement and prompt participants to execute the instructed configuration with the active hand. Participants were directed to produce and release each configuration as quickly as possible while keeping forces of passive fingers constant within resting range. To generate each configuration, a force exceeding $2.3 \mathrm{~N}$ had to be applied by active fingers (e.g., index and little; Fig. $2 A$ ) for $500 \mathrm{~ms}$ while passive fingers (e.g., thumb, middle, and ring) maintained a constant resting force. After successful configuration production, the baseline box turned red, which instructed participants to reduce all finger forces back to resting level. After configuration release, the baseline box turned gray again, and the next trial initiated after a $500 \mathrm{~ms}$ intertrial interval.

We predicted that repeated practice of a set of hand configurations would result in faster, more synchronous force production because of the formation of dedicated neural control modules encoding the learned synergies. We parameterized speed by execution time (ET) and reaction time (RT). RT was defined as the interval from presentation of the go signal until the moment any finger force exceeded resting range (which marked configuration initiation). ET was the period from configuration initiation to release (Fig. 2B). Note that configurations had to be correctly generated within $7 \mathrm{~s}$ or the program proceeded to the next trial and the ET was recorded as $7 \mathrm{~s}$.

Movement accuracy was quantified as follows. Finger forces (in Newtons) were normalized between 0 and 1 , where 0 corresponded to force levels during presentation of the go signal (i.e., the resting force) and 1 to the force threshold for active fingers $(2.3 \mathrm{~N})$. Because the instruction merely demanded forces exceeding $2.3 \mathrm{~N}$, any higher force applied was designated the value of 1 . Additionally, as long as forces exerted by passive fingers were within resting range, a value of 0 was assigned. The accuracy during each individual trial was assessed by evaluating the force trajectory in fivedimensional digit space, which started at the origin (trial initiation) and progressed to the desired configuration (configuration press; Fig. $2 C$, black line) and returned to the origin (configuration release; data not shown). If a configuration was generated with perfect simultaneity and no passive finger coactivation, the force trajectory corresponded to a straight line (Fig. 2C, green line) toward the goal configuration (red sphere). Contrastingly, sequential engagement of active fingers or involuntary coactivation of passive fingers caused deviation from this straight-line trajectory. We quantified accuracy as the Euclidian norm $(\|\ldots\|)$ between the produced force $(5 \times 1$ vector, $f_{t}$ ) and the projection of the produced force onto the straight-line trajectory to the target $(c)$. This distance was averaged over all time points from initiation to release to produce the "mean deviation":

$$
\text { mean deviation }=\sum_{t=1}^{T}\left\|f_{t}-\frac{c^{T} f_{t}}{\|c\|^{2}} \cdot c\right\| / T
$$

Although it is theoretically possible that participants could speed up configuration execution at the expense of mean deviation, we did not find evidence of a speed-accuracy tradeoff. Rather, mean deviation decreases consistently accompanied ET decreases.

Participants were made aware of their performance by feedback provided on the computer screen. For each individual trial, when ET and mean deviation values were in the 20th percentile of the performance of the previous day, participants received one point. Additionally, at the end of each run, average ET and mean deviation, as well as total points (which determined financial rewards above standard remuneration) were displayed.

Twelve configurations (six two-finger and six three-finger) of approximately equivalent difficulty were selected based on a pilot study. Each participant completed all 12 configurations during pretest and posttests, with eight trials per configuration per hand ( 96 trials total). Participants were assigned a set of two two-finger and two three-finger configurations for training from a pool of seven predesignated, difficulty-matched sets that were counterbalanced between tDCS and sham groups. Each training session consisted of 24 runs (all permutations of possible orderings), and each configuration was executed four times per run, yielding a total of 384 trials per day.

Sequence task. The sequence task required participants to press each finger in a predefined order, which was represented as numeric characters on a computer screen. For each trial, the numbers 1-5 were displayed in a specific series on the screen, with 1 corresponding to thumb and 5 to little finger (see Fig. $5 \mathrm{~A}$ ). Before trial initiation, there was a $2.7 \mathrm{~s}$ instruction phase during which the required sequence was explicitly shown on the screen. After presentation of the go signal (a set of five white asterisks), participants had to generate this sequence as quickly as possible from memory. Each active digit had to exert a force exceeding $0.6 \mathrm{~N}$, while passive fingers applied forces below $0.5 \mathrm{~N}$.

ET was calculated as the time from first key press to last key release for correct sequences only (see Fig. 5B), and error rate was defined as the percentage of trials with one or more incorrect presses. To measure performance on the same position of the speed-accuracy tradeoff function (Reis et al., 2009), accuracy demands were held constant across the experiment. We accomplished this by directing participants to increase ET (slow down) when error rate exceeded 20\% and decrease ET (speed up) when error rate was less.

Participants received one point for each correct sequence with ET in the 20th percentile of the performance of the previous day. Also, at the end of each run, participants were made aware of mean ET and error rate and total points (which determined monetary bonuses).

Twelve difficulty-matched five-finger sequences, which did not share any subsequential transitions longer than two presses, were selected based on a pilot study and a previous study (Wiestler and Diedrichsen, 2013). All sequences were performed during pretest and posttests, with eight trials per sequence per hand ( 96 trials total). During training, subjects were assigned four of these sequences from a pool of seven predefined sets, which were counterbalanced between stimulation groups. Each of the four sequences for training was practiced four consecutive times in 24 runs, which resulted in 384 trials per day.

Individuation task. When a person moves one finger, others tend to move involuntarily — a phenomenon known as "enslaving" or "interdependency" of fingers - which occurs as a result of mechanical and neural connections between digits on the same hand (Zatsiorsky et al., 1998, 2000; Schieber and Santello, 2004). Healthy participants generally can move one finger without coactivating others excessively, but patients suffering from cortical motor stroke often exhibit decreased individuation of paretic hand digits (Lang and Schieber, 2003, 2004; Li et al., 2003; Raghavan et al., 2006; for review, see Krakauer, 2005).

In the context of this study, we investigated individuation to ascertain whether any observed tDCS effects on synergy learning reflected general 

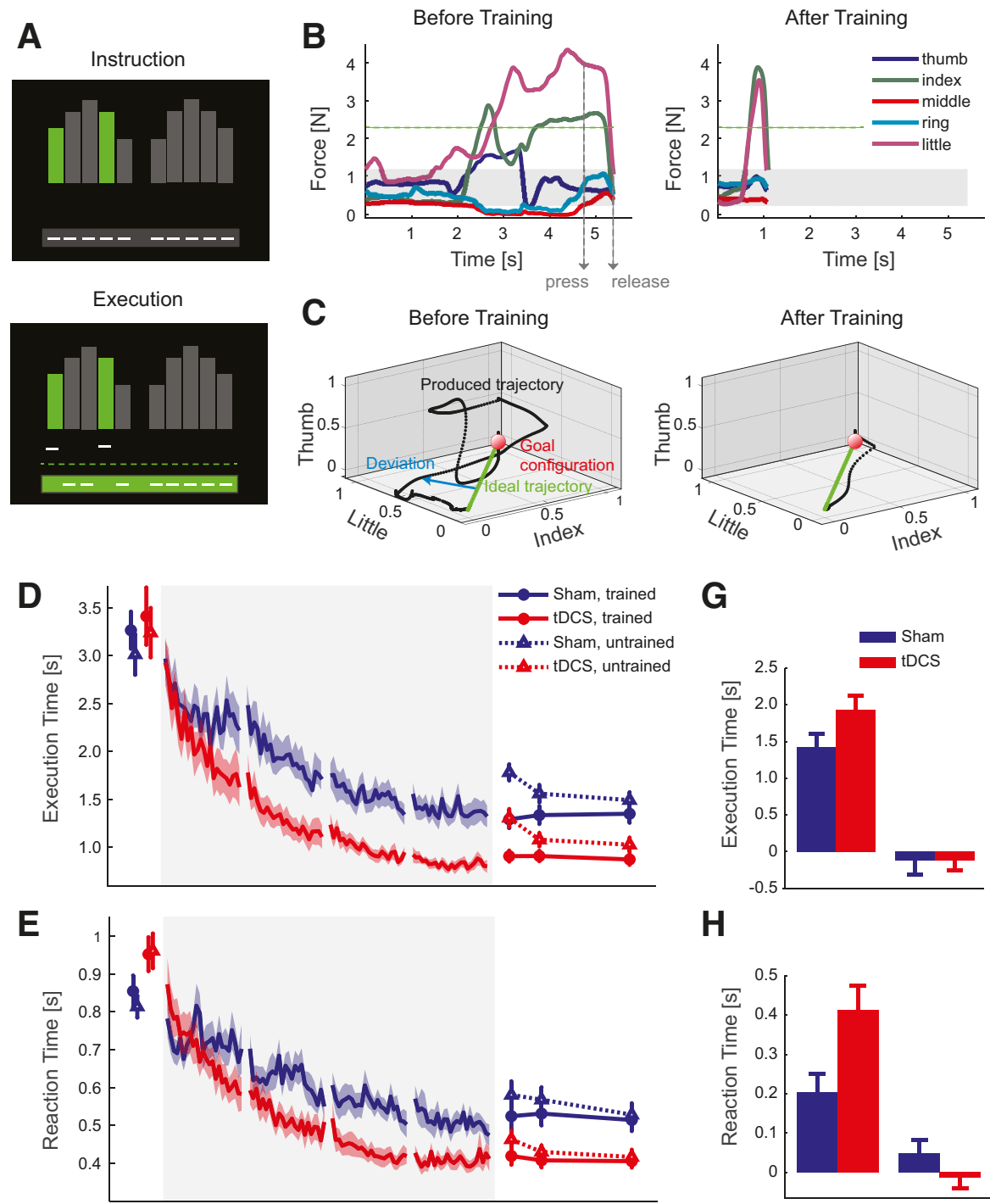

H
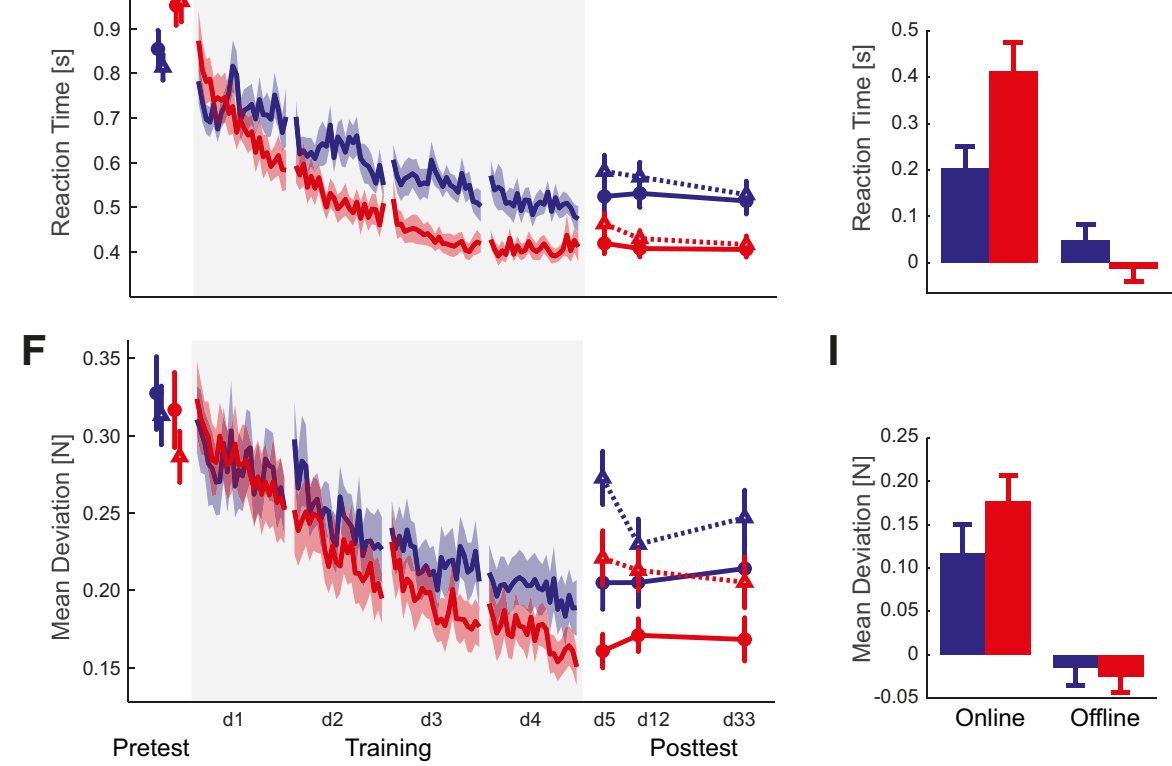

I

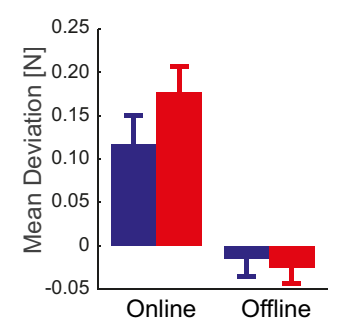

Figure 2. TDCS improves learning of hand muscle synergies for the trained left hand. $A$, Task design. In the instruction phase, participants generated a low, constant force with all fingers, such that all force cursors (white horizontal lines) were within the baseline box (gray horizontal box). The imperative signal (green vertical bars) specified active fingers (here, left hand index and little). In the execution phase, active fingers had to exert a force that exceeded the target level of $2.3 \mathrm{~N}$ (dashed green line), whereas forces of passive fingers remained within resting range (green horizontal box). $B$, Exemplar trials of a representative participant. Before training, forces exerted by all five fingers were sequentially adjusted until the required configuration was achieved. After training, active finger forces were generated with greater simultaneity and minimal coactivation of passive fingers. C, Corresponding force trajectories (black line) in a three-dimensional finger space consisting of two active fingers (index and little) and one passive digit (thumb). Ideal performance - simultaneous execution of the configuration without coactivation of passive fingers—-would constitute a straight-line trajectory (green line) to the goal configuration (red sphere). The deviation from this straight-line trajectory (blue arrow), averaged across the entire execution phase (initiation to release) - i.e., the mean deviation — was used to parameterize accuracy. Although only three finger dimensions are shown here, mean deviation was, in fact, calculated over all five dimensions. In the examples shown here, mean deviation was 0.32 before and 0.09 after training. D. Average ET of trained configurations (circle and solid line) in pretest (day 1), training (days 1-4), and posttests (days 5, 12, and 33) for sham (blue) and tDCS (red) groups. For pretest and posttest data, ET was averaged over the entire session, whereas, for training, it was averaged across each of the 24 runs per session. Eight untrained configurations (triangle and dashed line) were tested during pretest and posttests. $\boldsymbol{E}, \boldsymbol{F}$, Average RT $(\boldsymbol{E})$ and mean deviation $(\boldsymbol{F})$, in the same format. $\mathbf{G}-\boldsymbol{I}, 0$ nline and offline learning of ET $(\boldsymbol{G}), \mathrm{RT}(\boldsymbol{H})$, and mean deviation $(\boldsymbol{I})$ for sham and tDCS groups. Positive and negative values, respectively, signify performance improvement and decrement. Error bars and the shaded region of the training data indicate between-subject SEM. 
improvements in the control of hand muscles. In the individuation task, all digits of the active hand had to be pressed at a moderate baseline force $(2.15-3.45 \mathrm{~N})$ to begin each trial. The target forces of all fingers were represented as boxes with a height corresponding to $1.3 \mathrm{~N}$ on the screen, and, as with the configuration and sequence tasks, actual finger forces were manifested as white force cursors (see Fig. 4A). After fingers exerted the correct resting force arrangement for $500 \mathrm{~ms}$, one box turned green (which designated the active finger) and completed three positional changes, each lasting $0.66 \mathrm{~s}$ : it progressed upward to a height corresponding to a 10.8 $\mathrm{N}$ press, remained stationary at that height, and moved back to the original position. To maintain the force cursor inside this box, participants had to progressively increase force applied by the active finger to the target level and subsequently gradually decrease force back to resting level. While increasing and decreasing active finger forces, those exerted by passive fingers had to remain constant at resting threshold.

To parameterize performance, we used the deviation of each finger from target force to calculate root mean square error (RMSE), which served as a composite measure of active finger accuracy and passive digit coactivation. RMSE was presented on the screen after each trial. The individuation task was completed only during pretest and posttests and was not administered with $\mathrm{tDCS}$. Each run consisted of two trials per finger per hand, and there were five runs per session (i.e., 50 trials per hand).

\section{Data analyses}

We evaluated three aspects of the tDCS effect on synergy and sequence learning: training effects, durability, and generalization. The tDCS effect across the $4 \mathrm{~d}$ of training was evaluated using repeated-measures (RM) ANOVA, with the factors group (tDCS vs sham) and training day. Additionally, the tDCS-associated learning effect was dissected into online and offline components. Online learning can be defined as the difference between the first and final block of a session and offline learning as the difference between the final block of day $N-1$ and first block of day $N$. However, the beginning and end of individual training sessions might not be representative of overall session learning (e.g., as a result of warm-up decrement at the beginning or fatigue at the end); therefore, we implemented linear regression across individual training sessions to estimate skill parameters at initial and concluding runs and calculated online and offline learning using these adjusted values.

To assess the durability of the tDCS effect, participants were tested on 3 posttest days without the application of tDCS. Rather than statistically testing the difference between pretest and posttests, we analyzed posttest data using an analysis of covariance (ANCOVA), in which pretest data was used as a covariate. This method optimally accounts for interindividual differences in pretest performance without inflating the variability of the measures through the relatively high within-subject variability of pretest data. Note that, because participants were assigned to stimulation groups according to trained task pretest performance, no significant baseline differences were observed between tDCS and sham groups in task parameters (Table 1). Also, there were no significant pretest differences between the sham groups of sequence- and configuration-trained cohorts-this is important because these groups served as nontrained control groups for the respective other cohort (Table 1). We additionally used ANCOVA to evaluate intratask, intermanual, and intertask generalization (generalization to untrained configurations or sequences, untrained hand, and an untrained individuation task, respectively).

Data were analyzed using custom-written MATLAB routines. The threshold for statistical comparisons was $p<0.05$. All data presented in the text and figures are represented as mean \pm SEM.

\section{Results}

\section{Synergy learning}

\section{tDCS effects during training}

The first objective of this study was to determine whether tDCS affects muscle synergy learning and to evaluate the durability and generalization of observed effects. In the configuration task, participants had to rapidly and accurately generate complex, difficult patterns of muscular activation. We hypothesized that synergy learning would be characterized by faster execution (reduced ET), less motor planning (reduced RT), and more synchronous engagement of muscles (reduced mean deviation; see Materials and Methods).

When attempting configurations de novo during the pretest, participants tended to sequentially adjust forces on each digit to achieve the required configuration, which resulted in long ET (Fig. 2B, left) and large mean deviation (Fig. 2C, left). By the termination of training, a given configuration was generated as a functional unit, with the correct combination of fingers engaged synchronously, which resulted in short ET (Fig. $2 B$, right) and low mean deviation (Fig. $2 C$, right). To evaluate the effects of training and tDCS, we conducted an RM-ANOVA with the factors group (tDCS vs sham) and training day (days 1-4). The basic effect of training across the $4 \mathrm{~d}$ and across groups was highly significant for ET $\left(F_{(3,78)}=105.82, p<1 \mathrm{e}^{-10}\right.$; Fig. $\left.2 D\right)$, RT $\left(F_{(3,78)}=139.98, p<1 \mathrm{e}^{-10}\right.$; Fig. $\left.2 E\right)$, and mean deviation $\left(F_{(3,78)}=59.51, p<1 \mathrm{e}^{-10}\right.$; Fig. $\left.2 F\right)$. Therefore, after training, muscle engagement became faster and more synchronous.

On top of these training effects, tDCS led to improvement in execution and planning speeds for configurations. In the final training session, $\mathrm{tDCS}$ recipients exhibited $\sim 40 \%$ faster ET than sham (Fig. 2D). Across training days, there was a significant effect of $\operatorname{tDCS}\left(F_{(1,26)}=10.85, p=0.0029\right)$. Additionally, RT was $\sim 20 \%$ faster with tDCS (Fig. $2 E$ ), and there was a significant tDCS effect across training sessions $\left(F_{(1,26)}=5.65, p=0.025\right)$, as well as a tDCS $\times$ day interaction $\left(F_{(3,78)}=3.22, p=0.027\right)$.

Rather than reflecting facilitation of learning, these tDCSassociated speed advantages could be explained by a shifted speed-accuracy tradeoff, whereby participants achieved faster ETs and RTs by sacrificing accuracy. For example, fast ET could be achieved by making rapid sequential finger force adjustments, which would yield poor force synchrony. To determine whether tDCS elicited authentic skill improvements, we compared group mean deviations. In the final training session, tDCS recipients exhibited $14.5 \%$ lower mean deviation (Fig. $2 F$ ), although the main effect of $\mathrm{tDCS}$ across training days was not significant $\left(F_{(1,26)}=0.66, p=0.42\right)$. Hence, based on these data, we can conclude that the reported tDCS improvements in ET were not confounded by parallel accuracy decrements. However, in the posttests, a significant facilitatory tDCS effect on mean deviation emerged (see next section).

Finally, we tested whether tDCS increased learning by boosting performance gains achieved within training sessions (that is, through an online effect) or reducing forgetting between sessions (i.e., an offline effect). When applied over multiple sessions during training of pinch force sequencing, $\mathrm{tDCS}$ has been shown to mainly influence offline learning (Reis et al., 2009). In contrast, for the configuration task, we observed that tDCS-related ET and RT improvements over the 4 training days were effected mainly through online learning (respectively, $t_{(26)}=1.88$ and 2.73, $p=$ 0.072 and 0.011 ; Fig. $2 G, H)$ rather than an offline effect $\left(t_{(26)}=\right.$ 0.004 and $1.39, p=0.99$ and 0.18 ). Note that tDCS recipients also exhibited a nonsignificant tendency for increased online learning of mean deviation $\left(t_{(26)}=1.32, p=0.20\right.$; Fig. $\left.2 I\right)$. Across all parameters, it is clear that $\mathrm{tDCS}$-associated synergy learning advantages emerged primarily within, rather than between, training sessions.

\section{Durability of tDCS effects}

To determine whether tDCS effects outlasted the final day of training, participants were tested during three additional sessions on days 5, 12, and 33 without additional application of tDCS. ANCOVA (see Materials and Methods) was used to compare posttest performance of sham and tDCS groups for the trained configurations of the trained hand. Because between-group dif- 
A
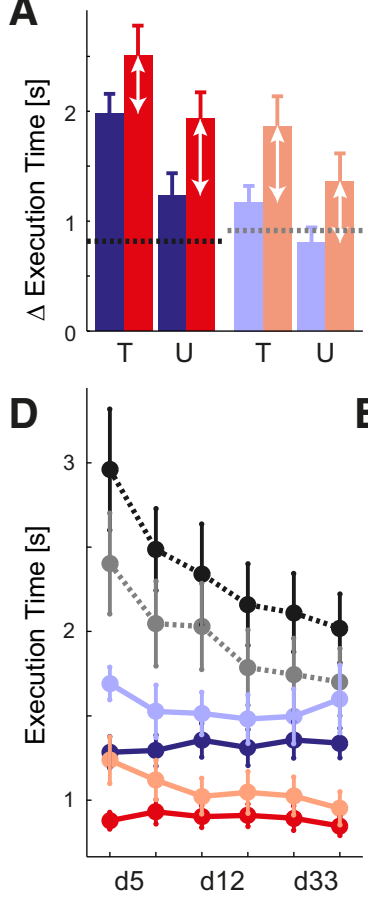

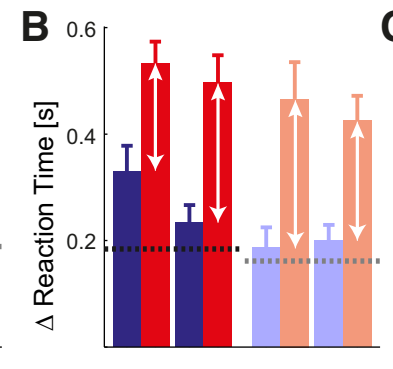

E

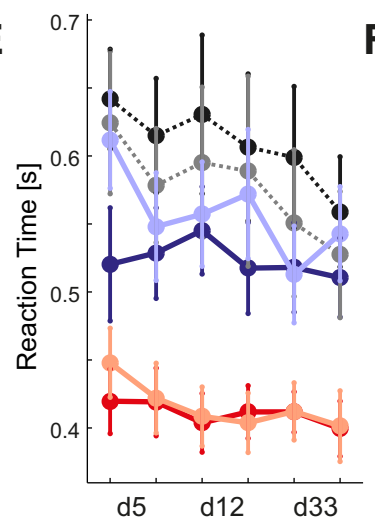

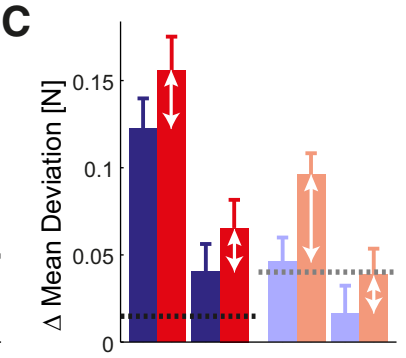

F

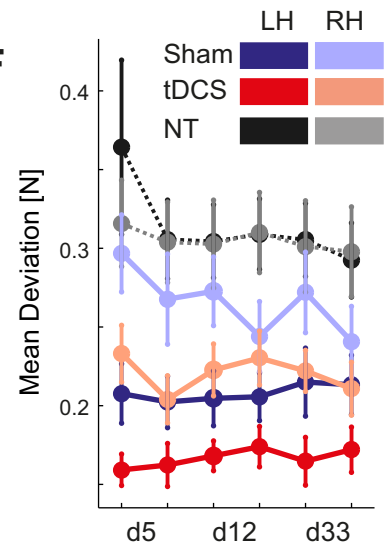

Figure 3. tDCS effects on synergy learning generalize to the untrained hand. $\boldsymbol{A}$, Average ET differences $(\Delta)$ between pretest (day 1) and first posttest (day 5) for trained $(\mathrm{T})$ and untrained $(U)$ configurations executed by the untrained right hand (RH; light blue/red) and trained left hand (LH; blue/red) for tDCS (red) and sham (blue) groups. tDCS effect sizes (white arrows) were similar across conditions. Dashed lines represent the ET difference for the nontrained (NT) group for the left (black) and right (gray) hands. $\boldsymbol{B}, \boldsymbol{C}$, Average RT (B) and mean deviation ( $\boldsymbol{C}$ ) differences, in the same format. $\boldsymbol{D}, \mathrm{ET}$ of trained configurations executed by trained and untrained hands across the three posttests. Each posttest day (d5, d12, and d33) is split into halves. $\boldsymbol{E}, \boldsymbol{F}, \mathrm{RT}(\boldsymbol{E})$ and mean deviation $(\boldsymbol{F})$, in the same format. Error bars indicate between-subject SEM.

ferences in ET did not change significantly across the three posttests (as reflected by lack of group $\times$ day interaction: $F_{(2,52)}=$ $0.726, p=0.489$; RM-ANOVA), comparisons were performed on averaged posttest performance.

The tDCS-associated advantages were fully preserved for 4 weeks after cessation of training (Fig. 2D-F, red circles/solid line): there were significant tDCS effects on $\operatorname{ET~}\left(F_{(1,25)}=24.56\right.$, $\left.p=4.18 \mathrm{e}^{-05}\right)$, RT $\left(F_{(1,25)}=14.30, p=8.68 \mathrm{e}^{-04}\right)$, and mean deviation $\left(F_{(1,25)}=6.02, p=0.021\right)$. In summary, synergy learning can be augmented by tDCS in a durable manner, outlasting stimulation by at least 4 weeks.

\section{Synergy generalization}

Generalization of tDCS effects to untrained configurations

We aimed to determine whether tDCS only improved the acquisition of motor memories for specific synergies or whether it also facilitated more general parts of the motor representations, which could be transferred to novel movements. Therefore, we tested whether learning gains attributable to tDCS generalized to untrained left-hand configurations (i.e., whether they exhibited intratask generalization). Because we wanted to ascertain immediate generalization, we limited group comparisons to the first (day 5) posttest to exclude the additional practice effects that occurred across the three posttests as a result of repeated testing.

For eight unpracticed configurations of the left hand, sham recipients exhibited large improvements from pretest to posttest (Figs. $2 D-F$, blue triangle/dashed line, $3 A-C$, blue bar). A considerable part of these improvements could be due to repeated testing on the untrained configurations during the posttest rather than actual gen- eralization. Therefore, we compared these learning gains with those of an active control, nontrained group (see Materials and Methods) - i.e., the sham group of the sequencetrained cohort, which was also tested on the configuration task during pretest and posttests (Fig. 3A-C, dashed black line). Compared with this nontrained baseline, intratask generalization in the sham group was modest. For untrained configurations, the sham group performed significantly better than the nontrained group for ET $\left(F_{(1,23)}=9.37, p=0.0055\right)$, but there were no significant differences in $\mathrm{RT}\left(F_{(1,23)}=\right.$ 1.605, $p=0.218)$ or mean deviation $\left(F_{(1,23)}=0.992, p=0.330\right)$. Further underscoring the specificity of the effect of training in the sham group, trained configurations were executed with faster ET $\left(t_{(13)}=\right.$ $\left.7.55, p=4.18 \mathrm{e}^{-06}\right)$, RT $\left(t_{(13)}=7.75, p=\right.$ $\left.3.16 \mathrm{e}^{-06}\right)$, and lower mean deviation $\left(t_{(13)}=5.96, p=4.72 \mathrm{e}^{-05}\right)$ relative to untrained configurations.

In contrast to the modest generalization in the sham group, the tDCS effect clearly transferred to untrained configurations. ANCOVA revealed a significant facilitatory tDCS effect on ET $\left(F_{(1,25)}=\right.$ 17.74, $\left.p=2.87 \mathrm{e}^{-04}\right)$ and $\operatorname{RT}\left(F_{(1,25)}=\right.$ $11.41, p=0.0024)$, as well as a trend for mean deviation $\left(F_{(1,25)}=3.19, p=0.086\right.$; Figs. $2 D-F, 3 A-C)$. Remarkably, the magnitude of the tDCS effect on ET (defined as the difference between sham and tDCS groups in respective pretest vs posttest differences) of untrained configurations $(0.48 \mathrm{~s})$ was similar to trained configurations $(0.38 \mathrm{~s})$ (Fig. $3 A$, white arrows), which was also the case for RT and mean deviation (Fig. $3 B, C$ ). The tDCS effect for trained and untrained configurations was, in fact, so similar that it did not significantly interact with configuration type $\left(F_{(1,26)}=1.08,1.06\right.$, and $0.25, p=$ $0.31,0.31$, and 0.62 for ET, RT, or mean deviation, respectively).

When expressing the pretest to posttest improvement in ET for untrained configurations as a percentage of that obtained for trained configurations - using the improvement of the nontrained group as a baseline-the sham group exhibited $35.8 \%$ generalization. However, this ratio increased to $66.2 \%$ for the tDCS group. These observations, along with the above, suggest that $\mathrm{tDCS}$ acted on the part of the motor memory that benefitted performance of untrained configurations (i.e., the nonspecific component).

\section{Generalization of tDCS effects to the untrained hand}

To ascertain whether tDCS effects on synergy learning were effector-specific, we examined performance of the untrained right hand after left-hand training. Untrained hand performance is of particular interest in the context of bihemispheric tDCS, which may enhance learning better than unilateral stimulation as a result of cathodal suppression of the ipsilateral hemisphere (Vines et al., 2008b). Assuming that intermanual transfer occurs (at least in part) during skill acquisition, such suppression during training could attenuate information transfer between hemispheres or the formation of effector-independent representations and im- 
pair subsequent performance of the hand contralateral to the cathode (i.e., the untrained hand).

We first established whether left-hand training led to performance gains for the untrained right hand over and above the improvements attributable to repeated testing (Fig. $3 A-C$ ). Relative to the nontrained group, sham participants performed trained configurations with the untrained hand significantly faster during the day 5 posttest $\left(F_{(1,23)}=5.99, p=\right.$ $0.022)$. However, there were no differences in RT or mean deviation $\left(F_{(23)}=0.289\right.$ and $0.096, p=0.60$ and 0.76 , respectively) or in any of the three parameters for untrained configurations $\left(\right.$ all $\left.F_{(23)}<1.541, p>0.23\right)$.

In contrast, tDCS effects generalized strongly to the untrained hand (Fig. 3A$C)$. Averaged over trained and untrained configurations, tDCS recipients performed with significantly better ET $\left(F_{(1,53)}=16.89\right.$, $\left.p=1.38 \mathrm{e}^{-04}\right)$, $\operatorname{RT}\left(F_{(1,53)}=30.39, p=\right.$ $\left.1.07 \mathrm{e}^{-06}\right)$, and mean deviation $\left(F_{(1,53)}=\right.$ $8.30, p=0.0057)$ relative to sham. As was the case with the trained left hand, the size of the tDCS effect was similar for trained and untrained configurations: there were no interactions of tDCS $\times$ configuration type for ET, RT, or mean deviation $\left(F_{(1,26)}=0.12,3.30\right.$, and $0.35, p=0.74,0.081$, and 0.56 , respectively; RM-ANOVA).

When expressing ET improvement for the untrained hand (averaged across trained/untrained configurations) as a percentage of the improvement on the trained hand (trained configurations only), the sham group showed only $6.6 \%$ generalization, after using the improvements of the nontrained group as a baseline. However, this ratio increased to $41.4 \%$ for the tDCS group. Note that the tDCS group also exhibited greater generalization than sham for RT (21.7 vs $81.5 \%$ ) and mean deviation (0 vs $19.3 \%)$. These results suggest that $\mathrm{tDCS}$ acted on the part of the motor memory that was effector-independent.

The development of untrained right-hand ET for trained configurations across the three posttests revealed an additional noteworthy observation (Fig. $3 D$, see $E$ and $F$ for RT and mean deviation): despite a significant difference between $\mathrm{tDCS}$ and $\operatorname{sham}\left(F_{(1,26)}=8.85\right.$, $p=0.006$; RM-ANOVA), the group $\times$ day interaction was not significant $\left(F_{(2,52)}=0.67, p=0.51\right)$ indicating that the untrained hand improved for both groups by a similar amount. In other words, the initial group difference was preserved over the course of the 3 posttest days as the performance of the untrained hand for each group approached that of the respective trained hand. Similar to the phenomenon in which previous motor memories facilitate relearning (Zarahn et al., 2008), effector-specific information for the trained hand may have served as a model to promote the learning of the untrained hand during the posttests. In contrast to immediate benefits on performance of the untrained hand, this finding may indicate the existence of "delayed" transfer (i.e., subsequent transfer attributable to additional practice effects across the posttest and/or the passage of time).

Generalization of tDCS effects to the untrained individuation task Our observations that $\mathrm{tDCS}$ effects generalized to untrained configurations and to the untrained hand could suggest that, rather

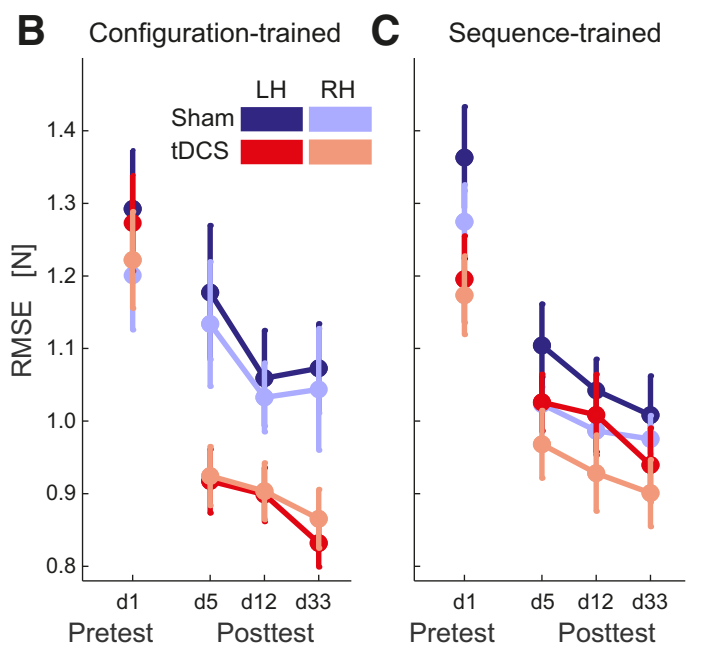

Figure 4. $\quad$ tDCS-coupled configuration training is associated with improved individuation. $\boldsymbol{A}$, Task design. In the instruc列 rest blue/red) and right hand ( $\mathrm{RH}$; light blue/red) for configuration-trained $(\boldsymbol{B})$ and sequence-trained $(\boldsymbol{C})$ cohorts. Error

than affecting the motor system per se, tDCS boosted capabilities in cognitive domains involved in motor skill learning (Dayan and Cohen, 2011). Curiously, when participants were instructed to semantically recall (write down) the trained configurations during the posttests, the tDCS group exhibited significantly better memory (Table 1), which could reflect enhanced cognitive processing or motor memory (or both). To test for genuine facilitation of motor synergy learning (which should improve general hand control), we examined performance on a finger individuation task that required participants to increase the force applied by one active finger to a high level without coactivating passive digits. This task required minimal attention and interpretation because it featured simple imperative stimuli that were easy to translate into action and demanded, rather than speed, precise hand control. If the reported tDCS effects on synergy learning were merely attributable to facilitation in cognitive domains, then tDCS and sham groups would be presumed to exhibit similar individuation. However, if tDCS increased the flexibility by which neural control modules could be recombined to produce novel hand shapes, this could lead to better hand control and, by extension, improved individuation.

Individuation accuracy was parameterized by RMSE between desired and produced finger forces - a measure that has been implemented by others to quantify enslaving (Raghavan et al., 2006). ANCOVA revealed a significant effect of tDCS (averaged across active and passive fingers and across posttests), such that tDCS recipients displayed $\sim 20 \%$ better posttest RMSE for left $\left(F_{(1,25)}=11.92, p=0.0020\right)$ and right $\left(F_{(1,25)}=11.20, p=\right.$ 0.0026 ) hands relative to sham (Fig. $4 B$ ). To further evaluate intertask transfer, we also compared performance on the sequence task: $\mathrm{tDCS}$ recipients exhibited significant ET advantages relative to sham on left and right hands $\left(F_{(1,25)}=6.830\right.$ and 9.234, $p=0.0149$ and 0.00550 , respectively; ANCOVA), without error rate differences $\left(F_{(1,25)}=0.146\right.$ and $0.233, p=0.706$ and 0.633$)$. Despite these preliminary results, a greater range of tasks is required to ascertain the generality of these supposed tDCS-related 
improvements in hand control (in particular whether they transfer to everyday activities).

To summarize, tDCS effects during configuration training transferred to unpracticed configurations and the untrained hand. Although the effect sizes of tDCS on all four types of configurations were similar, without additional experiments, we do not believe it can be concluded that tDCS only acted on the part of the motor memory that was transferrable (see Discussion). However, our results clearly indicate that tDCS did affect configuration nonspecific and effector-independent motor representations. Moreover, tDCS-related improvements also generalized to finger individuation and sequencing, implying that it acted on a part of the motor memory that was even transferrable to untrained tasks.

\section{Sequence learning}

\section{tDCS effects during training}

The second objective of this study was to compare tDCS effects on synergy learning and generalization with those observed with a finger sequence task. The sequence task required the successive production of five finger presses (Fig. $5 \mathrm{~A}$ ) and the parameter of interest was ET, while participants were instructed to keep the error rate at a constant level (see Materials and Methods). During the pretest, sequences were generated as five chronologically discrete presses, and, subsequent to training, presses became highly temporally overlapping (Fig. 5B).

RM-ANOVA with the factors group and training day revealed a substantial effect of training across the $4 \mathrm{~d}\left(F_{(3,78)}=115.17, p<\right.$ $1 \mathrm{e}^{-10}$; Fig. $5 C$ ). Beyond the basic training effect, tDCS recipients exhibited 37\% faster ET than sham on the final training day. Across training days, there was a significant tDCS effect on ET $\left(F_{(1,22)}=9.96, p=0.0046\right)$, as well as a tDCS $\times$ day interaction $\left(F_{(3,66)}=4.68, p=0.005\right)$, which reflected progressive betweengroup divergence. As intended, we observed stable error rates across training, which did not differ between groups $\left(F_{(1,22)}=\right.$ $0.63, p=0.44$; Fig. $5 D$ ).

The tDCS effect on ET occurred through an offline $\left(t_{(22)}=\right.$ $2.61, p=0.016)$, rather than online $\left(t_{(22)}=0.10, p=0.92\right)$, effect (Fig. 5E). Whereas sham recipients suffered significant offline skill decrement (overnight forgetting), tDCS recipients experienced slight offline gains, and both groups learned similarly within session. These results replicate previously documented offline tDCS effects (Reis et al., 2009; Tecchio et al., 2010; Kantak et al., 2012) but are opposite to what we observed for synergy learning.

\section{Durability of tDCS effects}

Both training- and tDCS-related ET advantages for trained sequences were preserved for at least 4 weeks after training (Fig. $5 C)$. Relative to sham, tDCS recipients executed trained sequences with significantly faster ET (averaged across posttests; $F_{(1,21)}=40.59, p=2.50 \mathrm{e}^{-06}$ ), without difference in error rate $\left(F_{(1,21)}=0.059, p=0.81\right)$. tDCS-related sequencing gains, like those exhibited with synergy learning, were durable.

\section{Sequence generalization}

Generalization of $t D C S$ effects to untrained sequences

In addition to evaluating tDCS effects on sequence training, we assessed generalization. As with the configuration task, we used the performance of a nontrained group (see Materials and Methods) to estimate how much of the performance gain from pretest to posttest was induced by repeated testing and how much was caused by generalization of left-hand training.
Even without tDCS, we observed evidence for substantial intratask transfer (Figs. 5C, 6A). Relative to the nontrained group, sham sequence-trained subjects exhibited significant ET benefits on the eight untrained sequences $\left(F_{(1,23)}=12.43, p=0.0018\right)$, without difference in error rate $\left(F_{(1,23)}=1.804, p=0.192\right)$. Despite this considerable generalization, in the sham group, trained sequences were performed with faster ET than untrained and with lower error rate $\left(t_{(11)}=9.57\right.$ and $2.72, p=1.14 \mathrm{e}^{-06}$ and 0.020 , respectively), indicating that learning was also, to a degree, sequence-specific.

As observed for the configuration task, the tDCS effect for trained sequences generalized to untrained sequences (Figs. $5 C$, $6 A)$. There was a significant tDCS effect on untrained sequences $\left(F_{(1,21)}=12.40, p=0.002\right)$ in the absence of error rate dissimilarities $\left(F_{(1,21)}=0.048, p=0.83\right)$. The size of the tDCS effects on ET of untrained $(0.35 \mathrm{~s})$ and trained $(0.47 \mathrm{~s})$ sequences were similar (Fig. $6 A$ ), and there was no tDCS $\times$ sequence type interaction $\left(F_{(1,22)}=1.57, p=0.22\right)$. When expressing the ET benefit for untrained sequences as a percentage of the gain for trained sequences using the improvements of the nontrained group as a baseline, we observed that this ratio increased from $51.5 \%$ for the sham to $65.6 \%$ for the tDCS group, suggesting that tDCS operated (at least in part) on the transferrable component of the motor memory, as was the case with synergy learning.

\section{Generalization of $t D C S$ effects to untrained hand}

Immediate intermanual generalization of training and tDCS effects for sequences also mostly mirrored those of synergies. We first compared performance of the sham group with the nontrained group to ascertain generalization (Fig. $6 \mathrm{~A}$ ) and observed significant improvements in ET of trained $\left(F_{(1,23)}=11.16, p=\right.$ $0.0028)$, as well as untrained $\left(F_{(1,23)}=11.18, p=0.0028\right)$ sequences of the untrained hand, without error rate differences $\left(F_{(1,23)}=0.13\right.$ and $0.14, p=0.72$ and 0.71 , respectively; Fig. $\left.6 B\right)$.

The tDCS effect additionally generalized to the untrained right hand (Fig. 6A). Averaged across trained and untrained sequences of the untrained hand, tDCS recipients performed with significantly faster ET than sham $\left(F_{(1,45)}=13.13, p=7.37 \mathrm{e}^{-04}\right.$; ANCOVA), without error rate difference $\left(F_{(1,45)}=0.081, p=\right.$ 0.78 ; Fig. $6 B)$. As was the case with the trained hand, the size of the tDCS effect for ET was similar for trained and untrained sequences, and there was no interaction of tDCS $\times$ sequence type $\left(F_{(1,22)}=0.584, p=0.309\right)$. Expressed as a percentage of the learning gain for trained sequences of the trained hand, the proportion of intermanual generalization increased from $38.6 \%$ for sham participants to $58.2 \%$ for the tDCS group, indicating that tDCS influenced effector-independent representations.

As with synergy learning, we observed evidence for delayed intermanual transfer of sequences: for trained sequences of the untrained hand, both groups progressively reached the ET achieved by the trained hand, which occurred with no confounding changes in error rate (Fig. $6 C, D)$. Despite a significant tDCS effect on untrained hand ET across posttests $\left(F_{(1,22)}=9.79, p=\right.$ 0.0049; RM-ANOVA), there was no tDCS $\times$ day interaction $\left(F_{(2,44)}=1.77, p=0.18\right)$, implying that both groups reached different asymptotic learning levels with the untrained right hand - a level that appeared to be set by that of the trained hand.

Generalization of tDCS effects to untrained individuation task Finally, we evaluated intertask generalization in the sequencetrained cohort. In the individuation task, tDCS and sham groups improved in RMSE at similar rates from pretest to posttests (Fig. $4 C)$. ANCOVA revealed no significant differences between groups for left or right hands $\left(F_{(1,21)}=0.36\right.$ and $0.26, p=0.56$ 
A

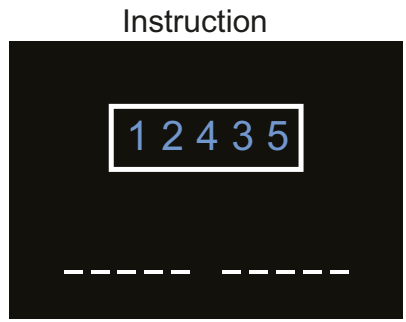

Execution

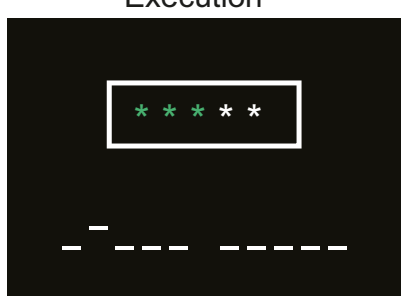

B

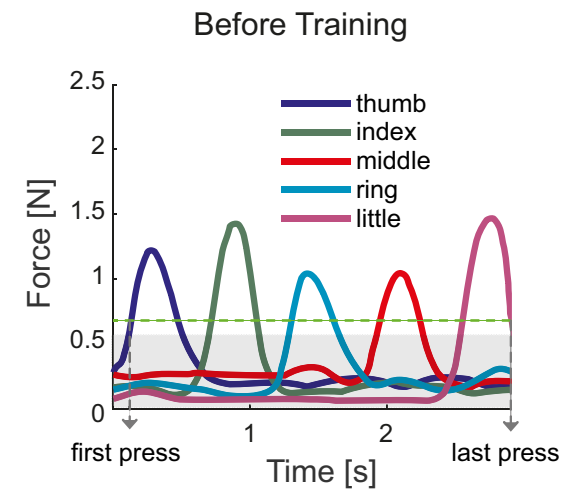

After Training

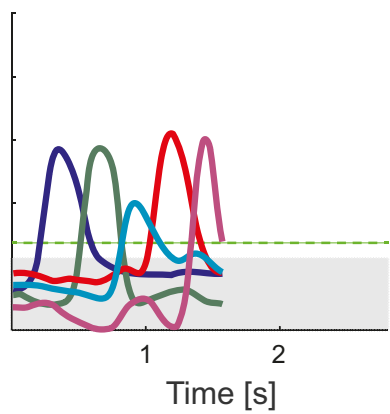

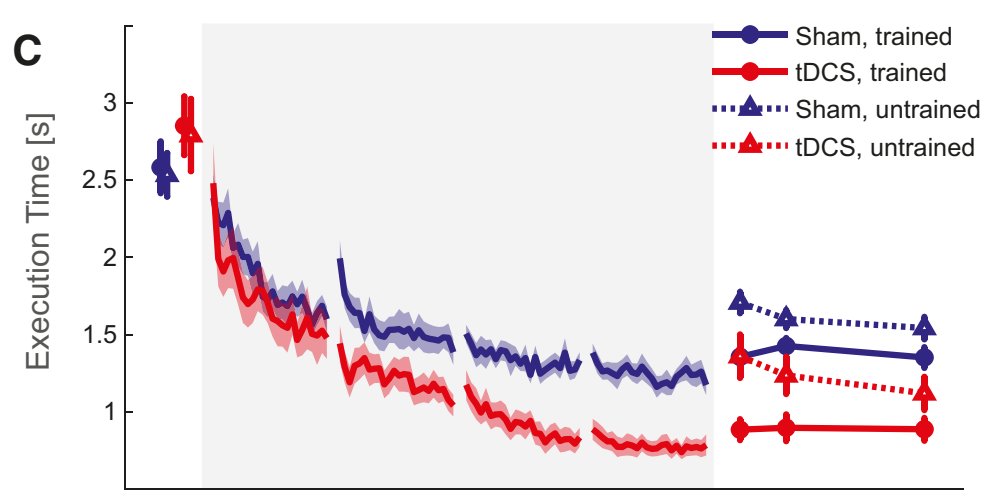

E
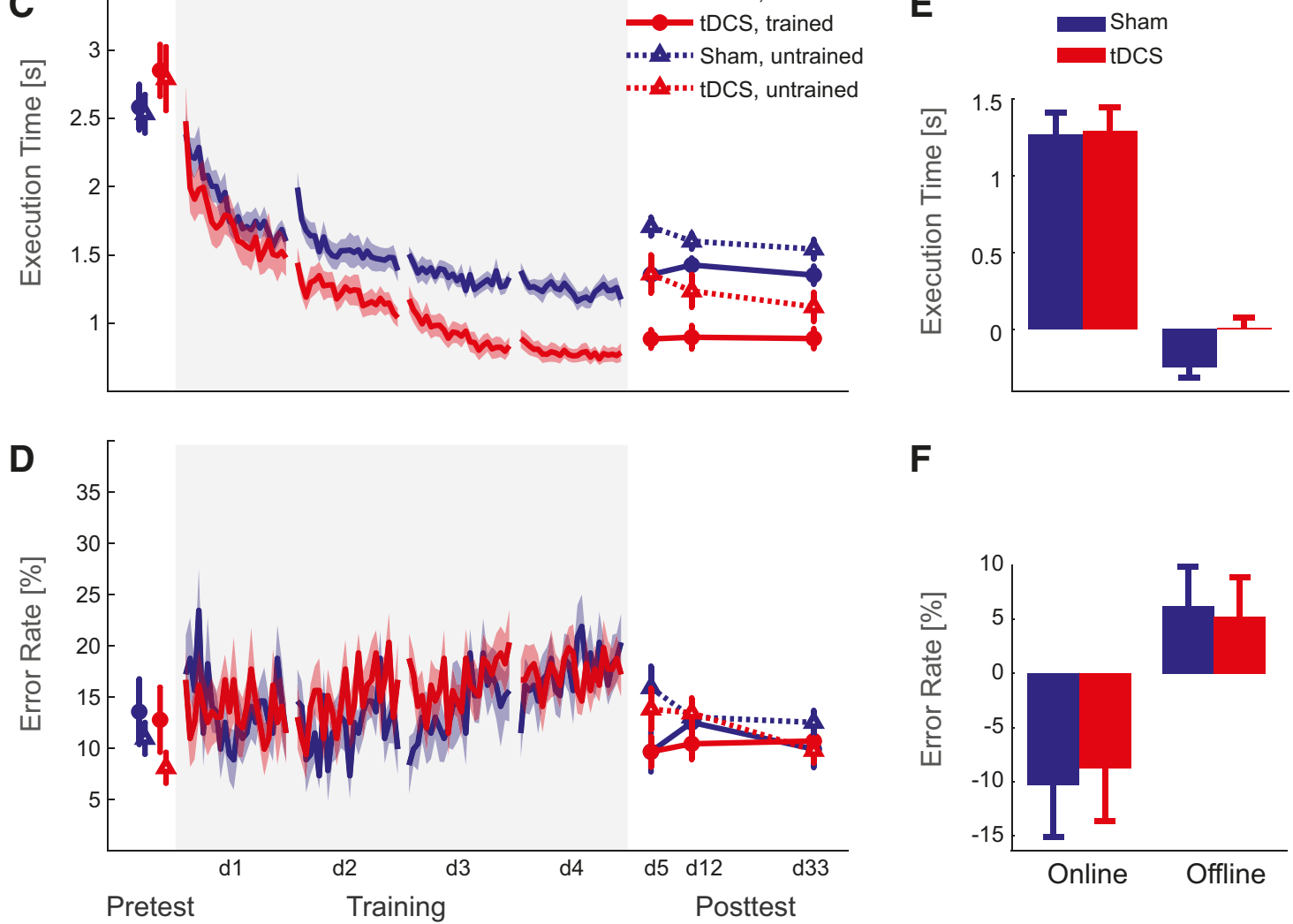

$\mathbf{F}$

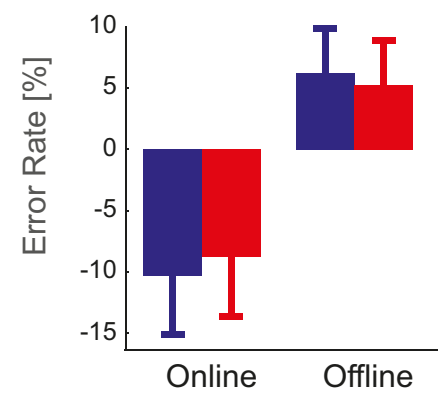

Figure 5. tDCS improves finger sequence learning for the trained left hand. $\boldsymbol{A}$, In the instruction phase, a sequence was displayed in blue numbers ( 1 for thumb and 5 for little finger). As a starting signal, five white asterisks were presented, which instructed participants to execute the previously shown sequence from memory. After each individual press, the corresponding asterisk changed color to indicate a correct (green) or incorrect (red) press, and, after a full sequence execution, a central asterisk turned green for a correct sequence, red if one or more errors were made, or blue if the sequence was correct but executed too slowly. $\boldsymbol{B}$, Example trials of a representative participant. Before training, finger presses were distinct; after training, the sequence was executed substantially faster, with greater temporal overlap between consecutive presses. C, Average ET in pretest (day 1), training (days 1-4), and posttests (days 5, 12, and 33) for sham (blue) and tDCS (red) groups. $\boldsymbol{D}$, Average error rate in the same format. $\boldsymbol{E}, \boldsymbol{F}$, Online and offline improvements in sequence task in $E T(\boldsymbol{E})$ and error rate $(\boldsymbol{F})$ for sham and tDCS groups. Positive and negative values, respectively, signify performance improvement and decrement. Error bars and the shaded region of the training data indicate between-subject SEM.

and 0.62 , respectively). However, these comparisons may be complicated by the fact that tDCS recipients tended to perform with lower RMSE at baseline on the individuation task and may have had less capacity for improvement. Therefore, we also tested individuation in an independent cohort of 14 sequence-trained participants who were well matched at baseline (data not shown). Confirming the results above, the tDCS group merely exhibited nonsignificant trends for RMSE improvement $\left(F_{(1,11)}=2.23\right.$ and
1.77, $p=0.16$ and 0.21 ). We also tested the sequence-trained cohort on the configuration task and observed no differences between tDCS and sham in left or right hands in terms of ET $\left(F_{(1,21)}=0.0958\right.$ and $1.659, p=0.760$ and 0.217 , respectively $)$ or mean deviation $\left(F_{(1,21)}=0.110\right.$ and $0.384, p=0.744$ and 0.542$)$, and similar results were observed for the aforementioned independent cohort $\left(\mathrm{ET}: F_{(1,11)}=1.279\right.$ and $1.730, p=0.282$ and 0.215 ; mean deviation: $F_{(1,11)}=2.124$ and $0.159, p=0.173$ and 0.698$)$. 

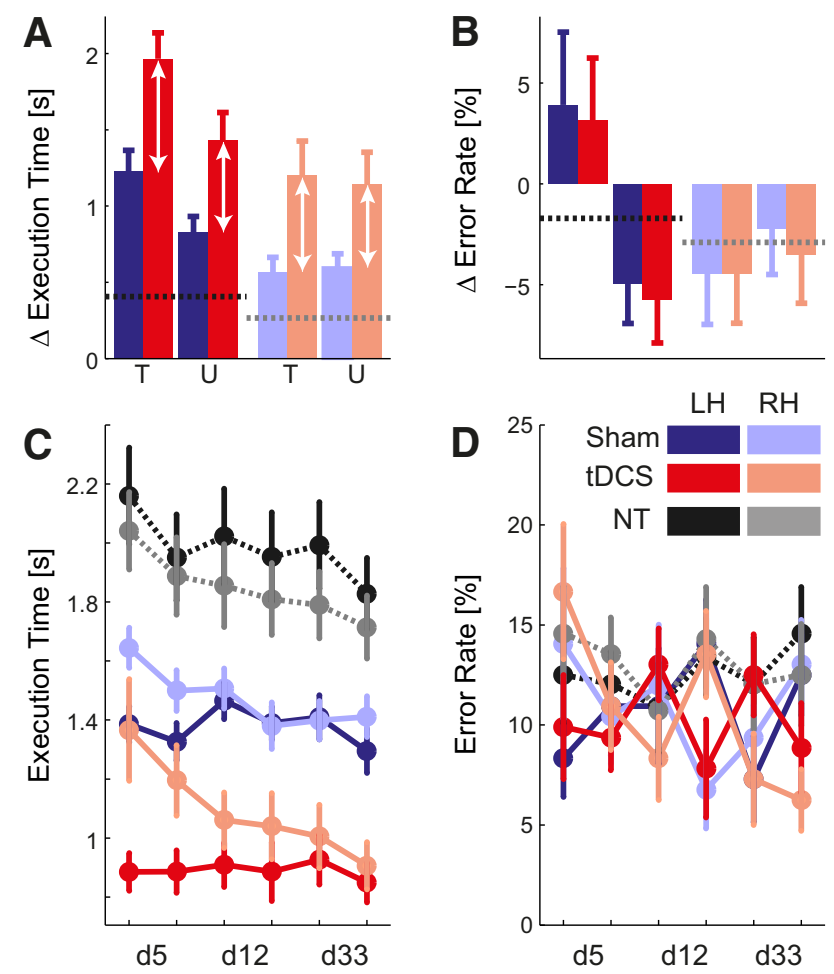

Figure 6. $\mathrm{tDCS}$ effects on sequence learning generalize to the untrained hand. $A, B$, Differences $(\Delta)$ between average pretest and day 5 posttest $E T(A)$ and error rate $(\boldsymbol{B})$ for trained $(\mathrm{T})$ and untrained $(\mathrm{U})$ sequences of the untrained right hand ( $\mathrm{RH}$; light red/blue) and trained left hand (LH; blue/red). tDCS effect sizes (white arrows) were similar across conditions. Dashed lines represent the ET difference for the nontrained (NT) group for the left (black) and right (gray) hands. $\boldsymbol{C}, \boldsymbol{D}$, Improvements in ET ( $\boldsymbol{C}$ ) and error rate $(\boldsymbol{D})$ of trained sequences executed by trained and untrained hands across the three posttests. Each posttest day is split into halves. Error bars indicate between-subject SEM.

Although the sequence task requires some degree of finger independence, application of tDCS during sequence training did not affect individuation task performance to the same degree as during configuration training. This supports our initial intuition that, in combination with $\mathrm{tDCS}$, tasks that specifically train synergy learning may improve hand control more and, by extension, may have larger benefit in stroke rehabilitation than motor sequencing tasks. However, it is possible that tDCS-associated benefits achieved with sequence training could transfer more to other tasks with sequential elements (e.g., typing). Additional testing with a range of tasks will be necessary to ascertain how tDCSinduced gains generalize to untrained motor skills.

In conclusion, tDCS effects on sequence learning and generalization mostly recapitulated those observed with synergies. For both synergy and sequence learning, bihemispheric tDCS of M1 elicited faster ET, without detriment to accuracy, and behavioral advantages achieved during training transferred broadly.

\section{Discussion}

Our primary aim was to characterize tDCS effects on synergy learning and generalization using a configuration task that required quick, synchronized production of difficult hand muscle activation patterns. Repeated-session bihemispheric tDCS of M1 led to increased speed (40\% faster ET and 20\% faster RT) and tendency for better force synchrony (lower mean deviation). During posttesting, even 1 month after cessation of stimulation, significant tDCS effects on both speed and synchrony were observed. This indicates that tDCS-coupled training promoted the acquisition of a durable motor memory rather than merely causing transient performance increases (Reis et al., 2009). The mechanism of this facilitation of plasticity is largely unknown: on one hand, tDCS could directly enhance synaptic plasticity, e.g., through processes that require BDNF secretion and TrkB activation (Fritsch et al., 2010). Alternatively, tDCS may facilitate performance during training, e.g., through reduction of inhibition (Stagg et al., 2009), and training at these higher performance levels may indirectly promote formation of a superior motor memory.

We also ascertained where in the motor hierarchy these effects occurred. We hypothesized that tDCS would facilitate the formation of neural populations that encode specific synergies. This proposal was motivated by the observation that musical training elicits experience-dependent changes in the modular architecture of M1 (Gentner et al., 2010). If tDCS increased plasticity in such circuits, poor generalization to unpracticed configurations would be expected. Indeed, tDCS could, theoretically, even impair performance of untrained configurations: considering that anodal tDCS removes local inhibition (Nitsche et al., 2005; Stagg et al., 2009; Tremblay et al., 2013), it could elicit spreading of cortical activation similar to a GABA antagonist (Schneider et al., 2002) and consequently reduce inhibition of muscles that should be deactivated (Sohn and Hallett, 2004; Beck and Hallett, 2011). Accordingly, sustained tDCS application could induce undesirable muscle coactivations, thereby hindering production of untrained configurations.

However, quite the converse was observed: tDCS recipients performed untrained configurations as fast as sham performed trained configurations (such that the tDCS effect size was similar for trained and untrained configurations). Moreover, tDCS recipients exhibited a greater proportion of transfer than sham. These observations suggest that tDCS acted on the transferrable part of the motor memory.

We additionally evaluated whether tDCS effects were effectorspecific. Although single-session bihemispheric tDCS has been shown to elicit greater behavioral improvements than unilateral stimulation in the effector contralateral to the anode (Vines et al., 2008b), consequences for the ipsilateral hand have, hitherto, not been examined. It is well established that $1 \mathrm{~mA}$ unihemispheric anodal tDCS increases (Nitsche and Paulus, 2000) whereas cathodal decreases (Nitsche et al., 2003) motor-evoked potentials (MEPs) at rest. Correspondingly, bihemispheric tDCS increases MEPs in anodal M1 and decreases MEPs in cathodal M1 (Williams et al., 2010; Mordillo-Mateos et al., 2012). Assuming that similar polarity-dependent changes in excitability occur during tDCS-coupled training, subsequent performance of the cathodeassociated hand could be compromised. Considering that unihemispheric cathodal tDCS has been associated with contralateral impairment (Vines et al., 2008a; Leite et al., 2011; Stagg et al., 2011), bihemispheric tDCS could adversely affect untrained hand performance. Although the untrained hand was never used during training-coupled tDCS, the cathode could inhibit information transfer and prevent formation of effector-independent representations. Nevertheless, we observed sizable immediate intermanual generalization of the tDCS advantage (as demonstrated by similar tDCS effect sizes for the trained and untrained hands and greater proportions of intermanual transfer in the tDCS group relative to sham). This indicates that tDCS also facilitated effector-independent components of motor memory during training, leading to a representation of motor skill that benefitted both hands. 
The strong intermanual transfer observed here could have been a consequence of the bihemispheric montage and may not extend to conventional unihemispheric tDCS: secondary motor areas that putatively represent movement skills in an effectorindependent manner, such as supplementary motor area (Perez et al., 2008), could have been targeted efficiently through the bihemispheric current, or the current flow may have changed the communication between motor cortices during training, possibly increasing the amount of mirrored activity in ipsilateral M1 (Diedrichsen et al., 2013). Additionally, high intermanual transfer could be attributable to cathodal enhancement of plasticity in the ipsilateral hemisphere: recent evidence suggests that cathodal unihemispheric tDCS at $2 \mathrm{~mA}$, the same intensity used in our study, actually increases MEPs (Batsikadze et al., 2013).

Although we provide clear evidence that tDCS acts on the transferrable part of the motor memory, the degree to which tDCS may have also enhanced synergy- and effector-specific components is difficult to evaluate. Simple pretest/posttest group differences in task parameters, as reported here, may not veridically reflect differences in skill. It could be argued, for example, that a $0.38 \mathrm{~s}$ improvement for trained configurations from $1.29 \mathrm{~s}$ (sham) to $0.91 \mathrm{~s}$ (tDCS) indicates a larger difference in skill level than a $0.48 \mathrm{~s}$ improvement for the untrained configurations from 1.78 to $1.31 \mathrm{~s}$, simply because, on a normal exponential learning curve, more training time would be required for the former change. Therefore, additional experiments that dissect transferrable and nontransferable components of motor learning are needed.

The second objective of this study was to evaluate bihemispheric $\mathrm{tDCS}$ effects on sequence learning and qualitatively compare them with our observations with synergy learning. We observed considerable enhancement of sequence learning (reflected by $37 \%$ faster ET), replicating previous observations with unihemispheric tDCS (Reis et al., 2009). The only difference between sequence and configuration tasks was the temporal dynamics with which tDCS improved ET. For synergy learning, advantages emerged within training sessions (i.e., through an online effect). However, with sequence learning, benefits ensued between sessions as a result of diminution in overnight forgetting (i.e., an offline effect), which is consistent with previous findings (Reis et al., 2009; Tecchio et al., 2010). Here, using identical training and stimulation protocols, we show that tDCS effects on online and offline learning are task dependent (Kantak et al., 2012).

Unfortunately, our results cannot reveal insight into the mechanisms underlying this task dependency. However, one possible explanation is that tDCS effects on plasticity vary with task demands. Online gains occur as a result of short-term, LTPlike plastic processes, which may saturate (Rioult-Pedotti et al., 2000) within individuation training sessions, causing performance to plateau. After training, labile learned material may be consolidated through protein synthesis (Luft et al., 2004; Peng and Li, 2009), returning short-term plasticity processes to baseline (Rioult-Pedotti et al., 2007). The degree to which tDCS increases capacity for short- and long-term plastic processes could be task dependent because different motor tasks vary in degree of short-term plasticity utilization and long-term forgetting (Dayan and Cohen, 2011). Indeed, configuration-trained sham recipients exhibited less overnight ET decay than sequence-trained sham recipients (Figs. $2 G, 5 E$ ).

As with synergy learning, tDCS-associated sequencing gains generalized to untrained sequences and the untrained hand. In addition to immediate intermanual generalization, we found evidence for delayed transfer: the untrained hand improved to the level attained by the trained hand, most likely through additional learning effects that occurred across posttests. Importantly, the untrained hand reached different levels of performance for sham and tDCS groups. These data suggest that effector- and configuration/sequence-specific knowledge for the trained hand may have served as a model for untrained hand learning. This implies that intermanual transfer may, in addition to being accessible to the untrained hand immediately after training, be induced by additional practice, similar to the way that previous motor memories can facilitate relearning (Zarahn et al., 2008).

tDCS is being explored as an adjunct to conventional neurorehabilitation (e.g., after stroke). For it to be beneficial, performance gains achieved during training should generalize to untrained motor tasks. Therefore, we determined whether tDCS-associated facilitation of synergy and sequence learning influenced digit individuation. tDCS recipients in the configuration-trained (but not sequence-trained) cohort exhibited substantial improvement in individuation. Moreover, configuration-trained tDCS recipients also exhibited improvements in sequence learning. Although these preliminary results may suggest that increasing synergy learning and/or execution through tDCS improves hand control, additional studies with a broader range of tasks are needed to ascertain the circumstances under which tDCS benefits transfer to untrained tasks.

Motor synergy learning might be more proximal to the processes that occur during stroke recovery than sequence learning. Rather than sequencing deficits (Hanlon, 1996; Pohl et al., 2001), a typical impairment associated with cortical motor lesions is severe loss of hand control, which has been demonstrated in humans (Lang and Schieber, 2003, 2004; Li et al., 2003; Krakauer, 2005; Raghavan et al., 2006) and, classically, in macaques (Lawrence and Kuypers, 1968). Such impairments may be due to aberrant, fractionated, merged, or reduced muscle synergies (Li et al., 2008; Clark et al., 2010; Gizzi et al., 2011; Cheung et al., 2012; Roh et al., 2013). In stroke patients, robot-assisted paretic arm training gains have been demonstrated to generalize to an untrained arm/elbow individuation task (Dipietro et al., 2007), illustrating that abnormal synergies can, in practice, be retuned. Our data show for the first time that, in healthy participants, tDCS enhances synergy learning in a transferrable and enduring manner-two essential characteristics of effective rehabilitative methods (Krakauer, 2006) — which suggests that tDCS might be a useful means to expedite or amplify synergy recovery in stroke.

\section{References}

Alonzo A, Brassil J, Taylor JL, Martin D, Loo CK (2012) Daily transcranial direct current stimulation (tDCS) leads to greater increases in cortical excitability than second daily transcranial direct current stimulation. Brain Stimul 5:208-213. CrossRef Medline

Ambrus GG, Al-Moyed H, Chaieb L, Sarp L, Antal A, Paulus W (2012) The fade-in-short stimulation-fade out approach to sham tDCS_reliable at 1 $\mathrm{mA}$ for naive and experienced subjects, but not investigators. Brain Stimul 5:499-504. CrossRef Medline

Batsikadze G, Moliadze V, Paulus W, Kuo MF, Nitsche MA (2013) Partially non-linear stimulation intensity-dependent effects of direct current stimulation on motor cortex excitability in humans. J Physiol 591:1987-2000. CrossRef Medline

Beck S, Hallett M (2011) Surround inhibition in the motor system. Exp Brain Res 210:165-172. CrossRef Medline

Bizzi E, D'Avella A, Saltiel P, Tresch M (2002) Modular organization of spinal motor systems. Neuroscientist 8:437-442. CrossRef Medline

Boggio PS, Nunes A, Rigonatti SP, Nitsche MA, Pascual-Leone A, Fregni F (2007) Repeated sessions of noninvasive brain DC stimulation is associated with motor function improvement in stroke patients. Restor Neurol Neurosci 25:123-129. Medline

Boroojerdi B, Foltys H, Krings T, Spetzger U, Thron A, Töpper R (1999) Localization of the motor hand area using transcranial magnetic stimula- 
tion and functional magnetic resonance imaging. Clin Neurophysiol 110: 699-704. CrossRef Medline

Brunoni AR, Schestatsky P, Lotufo PA, Bensenor IM, Fregni F (2013) Comparison of blinding effectiveness between sham tDCS and placebo sertraline in a 6-week major depression randomized clinical trial. Clin Neurophysiol. Advance online publication. Retrieved December 3, 2013. doi:10.1016/j.clinph.2013.07.020. CrossRef Medline

Cheung VC, Turolla A, Agostini M, Silvoni S, Bennis C, Kasi P, Paganoni S, Bonato P, Bizzi E (2012) Muscle synergy patterns as physiological markers of motor cortical damage. Proc Natl Acad Sci U S A 109:14652-14656. CrossRef Medline

Clark DJ, Ting LH, Zajac FE, Neptune RR, Kautz SA (2010) Merging of healthy motor modules predicts reduced locomotor performance and muscle coordination complexity post-stroke. J Neurophysiol 103:844857. CrossRef Medline

Dayan E, Cohen LG (2011) Neuroplasticity subserving motor skill learning. Neuron 72:443-454. CrossRef Medline

Diedrichsen J, Shadmehr R, Ivry RB (2010) The coordination of movement: optimal feedback control and beyond. Trends Cogn Sci 14:31-39. CrossRef Medline

Diedrichsen J, Wiestler T, Krakauer JW (2013) Two distinct ipsilateral cortical representations for individuated finger movements. Cereb Cortex 23:1362-1377. CrossRef Medline

Dipietro L, Krebs HI, Fasoli SE, Volpe BT, Stein J, Bever C, Hogan N (2007) Changing motor synergies in chronic stroke. J Neurophysiol 98:757-768. CrossRef Medline

Fritsch B, Reis J, Martinowich K, Schambra HM, Ji Y, Cohen LG, Lu B (2010) Direct current stimulation promotes BDNF-dependent synaptic plasticity: potential implications for motor learning. Neuron 66:198-204. CrossRef Medline

Gandiga PC, Hummel FC, Cohen LG (2006) Transcranial DC stimulation (tDCS): a tool for double-blind sham-controlled clinical studies in brain stimulation. Clin Neurophysiol 117:845-850. CrossRef Medline

Gentner R, Classen J (2006) Modular organization of finger movements by the human central nervous system. Neuron 52:731-742. CrossRef Medline

Gentner R, Gorges S, Weise D, Aufm Kampe K, Buttmann M, Classen J (2010) Encoding of motor skill in the corticomuscular system of musicians. Curr Biol 20:1869-1874. CrossRef Medline

Gizzi L, Nielsen JF, Felici F, Ivanenko YP, Farina D (2011) Impulses of activation but not motor modules are preserved in the locomotion of subacute stroke patients. J Neurophysiol 106:202-210. CrossRef Medline

Hanlon RE (1996) Motor learning following unilateral stroke. Arch Phys Med Rehabil 77:811-815. CrossRef Medline

Hardwick RM, Rottschy C, Miall RC, Eickhoff SB (2013) A quantitative meta-analysis and review of motor learning in the human brain. Neuroimage 67:283-297. CrossRef Medline

Kantak SS, Mummidisetty CK, Stinear JW (2012) Primary motor and premotor cortex in implicit sequence learning-evidence for competition between implicit and explicit human motor memory systems. Eur J Neurosci 36:2710-2715. CrossRef Medline

Kessler SK, Turkeltaub PE, Benson JG, Hamilton RH (2012) Differences in the experience of active and sham transcranial direct current stimulation. Brain Stimul 5:155-162. CrossRef Medline

Krakauer JW (2005) Arm function after stroke: from physiology to recovery. Semin Neurol 25:384-395. CrossRef Medline

Krakauer JW (2006) Motor learning: its relevance to stroke recovery and neurorehabilitation. Curr Opin Neurol 19:84-90. CrossRef Medline

Lang CE, Schieber MH (2003) Differential impairment of individuated finger movements in humans after damage to the motor cortex or the corticospinal tract. J Neurophysiol 90:1160-1170. CrossRef Medline

Lang CE, Schieber MH (2004) Reduced muscle selectivity during individuated finger movements in humans after damage to the motor cortex or corticospinal tract. J Neurophysiol 91:1722-1733. CrossRef Medline

Lawrence DG, Kuypers HG (1968) The functional organization of the motor system in the monkey. I. The effects of bilateral pyramidal lesions. Brain 91:1-14. CrossRef Medline

Leite J, Carvalho S, Fregni F, Gonçalves ÓF (2011) Task-specific effects of tDCS-induced cortical excitability changes on cognitive and motor sequence set shifting performance. PLoS One 6:e24140. CrossRef Medline

Li J, Wang ZJ, Eng JJ, McKeown MJ (2008) Bayesian network modeling for discovering "dependent synergies" among muscles in reaching movements. IEEE Trans Biomed Eng 55:298-310. CrossRef Medline

Li S, Latash ML, Yue GH, Siemionow V, Sahgal V (2003) The effects of stroke and age on finger interaction in multi-finger force production tasks. Clin Neurophysiol 114:1646-1655. CrossRef Medline

Luft AR, Buitrago MM, Ringer T, Dichgans J, Schulz JB (2004) Motor skill learning depends on protein synthesis in motor cortex after training. J Neurosci 24:6515-6520. CrossRef Medline

Mordillo-Mateos L, Turpin-Fenoll L, Millán-Pascual J, Núñez-Perez N, Panyavin I, Gómez-Arguelles JM, Botia-Paniagua E, Foffani G, Lang N, Oliviero A (2012) Effects of simultaneous bilateral tDCS of the human motor cortex. Brain Stimul 5:214-222. CrossRef Medline

Nitsche MA, Paulus W (2000) Excitability changes induced in the human motor cortex by weak transcranial direct current stimulation. J Physiol 527:633-639. CrossRef Medline

Nitsche MA, Paulus W (2001) Sustained excitability elevations induced by transcranial DC motor cortex stimulation in humans. Neurology 57: 1899-1901. CrossRef Medline

Nitsche MA, Nitsche MS, Klein CC, Tergau F, Rothwell JC, Paulus W (2003) Level of action of cathodal DC polarisation induced inhibition of the human motor cortex. Clin Neurophysiol 114:600-604. CrossRef Medline

Nitsche MA, Seeber A, Frommann K, Klein CC, Rochford C, Nitsche MS, Fricke K, Liebetanz D, Lang N, Antal A, Paulus W, Tergau F (2005) Modulating parameters of excitability during and after transcranial direct current stimulation of the human motor cortex. J Physiol 568:291-303. CrossRef Medline

O'Connell NE, Cossar J, Marston L, Wand BM, Bunce D, Moseley GL, De Souza LH (2012) Rethinking clinical trials of transcranial direct current stimulation: participant and assessor blinding is inadequate at intensities of 2mA. PLoS One 7:e47514. CrossRef Medline

Oldfield RC (1971) The assessment and analysis of handedness: the Edinburgh inventory. Neuropsychologia 9:97-113. CrossRef Medline

Overduin SA, d'Avella A, Carmena JM, Bizzi E (2012) Microstimulation activates a handful of muscle synergies. Neuron 76:1071-1077. CrossRef Medline

Palm U, Reisinger E, Keeser D, Kuo MF, Pogarell O, Leicht G, Mulert C, Nitsche MA, Padberg F (2013) Evaluation of sham transcranial direct current stimulation for randomized, placebo-controlled clinical trials. Brain Stimul 6:690-695. CrossRef Medline

Peng JY, Li BM (2009) Protein synthesis is essential not only for consolidation but also for maintenance and post-retrieval reconsolidation of acrobatic motor skill in rats. Mol Brain 2:12. CrossRef Medline

Penhune VB, Steele CJ (2012) Parallel contributions of cerebellar, striatal and M1 mechanisms to motor sequence learning. Behav Brain Res 226: 579-591. CrossRef Medline

Perez MA, Tanaka S, Wise SP, Willingham DT, Cohen LG (2008) Timespecific contribution of the supplementary motor area to intermanual transfer of procedural knowledge. J Neurosci 28:9664-9669. CrossRef Medline

Plazier M, Joos K, Vanneste S, Ost J, De Ridder D (2012) Bifrontal and bioccipital transcranial direct current stimulation (tDCS) does not induce mood changes in healthy volunteers: a placebo controlled study. Brain Stimul 5:454-461. CrossRef Medline

Pohl PS, McDowd JM, Filion DL, Richards LG, Stiers W (2001) Implicit learning of a perceptual-motor skill after stroke. Phys Ther 81:1780-1789. Medline

Priori A, Berardelli A, Rona S, Accornero N, Manfredi M (1998) Polarization of the human motor cortex through the scalp. Neuroreport 9:22572260. CrossRef Medline

Raghavan P, Petra E, Krakauer JW, Gordon AM (2006) Patterns of impairment in digit independence after subcortical stroke. J Neurophysiol 95: 369-378. Medline

Reis J, Fritsch B (2011) Modulation of motor performance and motor learning by transcranial direct current stimulation. Curr Opin Neurol 24:590596. CrossRef Medline

Reis J, Schambra HM, Cohen LG, Buch ER, Fritsch B, Zarahn E, Celnik PA, Krakauer JW (2009) Noninvasive cortical stimulation enhances motor skill acquisition over multiple days through an effect on consolidation. Proc Natl Acad Sci U S A 106:1590-1595. CrossRef Medline

Rioult-Pedotti MS, Friedman D, Donoghue JP (2000) Learning-induced LTP in neocortex. Science 290:533-536. CrossRef Medline

Rioult-Pedotti MS, Donoghue JP, Dunaevsky A (2007) Plasticity of the syn- 
aptic modification range. J Neurophysiol 98:3688-3695. CrossRef Medline

Roh J, Rymer WZ, Perreault EJ, Yoo SB, Beer RF (2013) Alterations in upper limb muscle synergy structure in chronic stroke survivors. J Neurophysiol 109:768-781. CrossRef Medline

Russo R, Wallace D, Fitzgerald PB, Cooper NR (2013) Perception of comfort during active and sham transcranial direct current stimulation: a double blind study. Brain Stimul 6:946-951. CrossRef Medline

Schieber MH, Santello M (2004) Hand function: peripheral and central constraints on performance. J Appl Physiol 96:2293-2300. CrossRef Medline

Schneider C, Devanne H, Lavoie BA, Capaday C (2002) Neural mechanisms involved in the functional linking of motor cortical points. Exp Brain Res 146:86-94. CrossRef Medline

Sherrington CS (1910) Flexion-reflex of the limb, crossed extension-reflex, and reflex stepping and standing. J Physiol 40:28-121. Medline

Sohn YH, Hallett M (2004) Surround inhibition in human motor system. Exp Brain Res 158:397-404. Medline

Stagg CJ, Best JG, Stephenson MC, O’Shea J, Wylezinska M, Kincses ZT, Morris PG, Matthews PM, Johansen-Berg H (2009) Polarity-sensitive modulation of cortical neurotransmitters by transcranial stimulation. J Neurosci 29:5202-5206. CrossRef Medline

Stagg CJ, Jayaram G, Pastor D, Kincses ZT, Matthews PM, Johansen-Berg H (2011) Polarity and timing-dependent effects of transcranial direct current stimulation in explicit motor learning. Neuropsychologia 49:800804. CrossRef Medline

Steele CJ, Scholz J, Douaud G, Johansen-Berg H, Penhune VB (2012) Structural correlates of skilled performance on a motor sequence task. Front Hum Neurosci 6:289. CrossRef Medline

Tecchio F, Zappasodi F, Assenza G, Tombini M, Vollaro S, Barbati G, Rossini PM (2010) Anodal transcranial direct current stimulation enhances procedural consolidation. J Neurophysiol 104:1134-1140. CrossRef Medline

Tremblay S, Beaulé V, Lepage JF, Théoret H (2013) Anodal transcranial direct current stimulation modulates GABAB-related intracortical inhi- bition in the M1 of healthy individuals. Neuroreport 24:46-50. CrossRef Medline

Tresch MC, Jarc A (2009) The case for and against muscle synergies. Curr Opin Neurobiol 19:601-607. CrossRef Medline

Vines BW, Nair D, Schlaug G (2008a) Modulating activity in the motor cortex affects performance for the two hands differently depending upon which hemisphere is stimulated. Eur J Neurosci 28:1667-1673. CrossRef Medline

Vines BW, Cerruti C, Schlaug G (2008b) Dual-hemisphere tDCS facilitates greater improvements for healthy subjects' non-dominant hand compared to uni-hemisphere stimulation. BMC Neurosci 9:103. CrossRef Medline

Watson D, Clark LA, Tellegen A (1988) Development and validation of brief measures of positive and negative affect: the PANAS scales. J Pers Soc Psychol 54:1063-1070. CrossRef Medline

Wiestler T, Diedrichsen J (2013) Skill learning strengthens cortical representations of motor sequences. eLife 2:e00801. CrossRef Medline

Wiestler T, McGonigle DJ, Diedrichsen J (2011) Integration of sensory and motor representations of single fingers in the human cerebellum. J Neurophysiol 105:3042-3053. CrossRef Medline

Williams JA, Pascual-Leone A, Fregni F (2010) Interhemispheric modulation induced by cortical stimulation and motor training. Phys Ther 90: 398-410. CrossRef Medline

World Medical Association (2002) World Medical Association Declaration of Helsinki: ethical principles for medical research involving human subjects. J Postgrad Med 48:206-208. Medline

Zarahn E, Weston GD, Liang J, Mazzoni P, Krakauer JW (2008) Explaining savings for visuomotor adaptation: linear time-invariant state-space models are not sufficient. J Neurophysiol 100:2537-2548. CrossRef Medline

Zatsiorsky VM, Li ZM, Latash ML (1998) Coordinated force production in multi-finger tasks: finger interaction and neural network modeling. Biol Cybern 79:139-150. CrossRef Medline

Zatsiorsky VM, Li ZM, Latash ML (2000) Enslaving effects in multi-finger force production. Exp Brain Res 131:187-195. CrossRef Medline 